\title{
QUADRATIC VARIATIONAL THEORY AND LINEAR ELLIPTIC PARTIAL DIFFERENTIAL EQUATIONS( $\left.{ }^{(}\right)$
}

\author{
BY \\ MAGNUS R. HESTENES
}

1. Introduction. One of the basic chapters in the calculus of variations is the study of integrals that are quadratic in the dependent functions. The Euler equations for such in tegrals are self-adjoint linear differential equations. The study of the second variation of a typical variational problem is essentially the study of quadratic integrals of this type. Simple integral problems lead to the study of ordinary differential equations and multiple integral problems lead to study of partial differential equations.

The study of quadratic functionals has always played an important role in the calculus of variations. However those arising from the various variational problems normally have been treated separately. The author has been convinced for many years that Hilbert space methods can be used to obtain a unified theory for these problems. In 1951 the author published a paper $[11]\left({ }^{2}\right)$ giving the fundamental theorems on quadratic forms in Hilbert space that are pertinent to problems in the calculus of variations. As is to be expected the quadratic forms that are of interest in variational theory have certain special properties. The most important class is comprised of those forms that can be made positive definite by the addition of a suitably chosen completely continuous quadratic form. The author called these Legendre forms, because in the applications to the calculus of variations these forms are characterized by the condition of Legendre in its strengthened form. In the theory of partial differential equations Gaarding's inequality [8] is equivalent to the statement that a certain quadratic form is a Legendre form. The coercieve integrodifferential forms of Aronszajn [1] are also Legendre forms. The second important class of quadratic forms are those that are lower semicontinuous with respect to weakly convergent sequences. These are the ones that can be made nonnegative by the addition of a completely continuous quadratic form. In variational theory they are normally characterized by the Legendre condition in its weaker form.

In addition to the study of Legendre forms and weakly lower semi-continuous forms, the author studied a theory of indices of these forms and a

Received by the editors February 28, 1961.

(') The preparation of this paper was sponsored by the Office of Naval Research and the Office of Ordnance Research, U. S. Army. Reproduction in whole or in part is permitted for any purpose of the United States Government.

A first draft of the paper was written while the author was a Guggenheim Fellow, 19541955, and while the author held a Fulbright Fellowship in Norway, 1954-1955.

(2) Numbers in brackets refer to the bibliography at the end of this paper. 
generalized theory of focal points. These are concerned with boundary value problems and Sturm Liouville theory. A sketch of the application of this theory to simple integral problems and ordinary differential equations was given. The application to multiple integral problems and to partial differential equations was postponed, except for some minor remarks. However, certain applications to multiple integral problems were kept in mind in the formulation of the theory.

One of the reasons for omitting the applications to multiple integral problems in the earlier paper was that a suitable condition of Legendre for multiple integral problems had not been completely established. In view of the results given by Graves [9] and Van Hove [24] the condition of Legendre at interior points of the region was essentially known for integrals involving derivatives of the first order. The corresponding results for integrals involving higher derivatives is a corollary of analogous results in the theory of partial differential equations developed by Gaarding, Friedrichs, Browder, and others. A suitable condition of Legendre at boundary points has not been established in the general case. If the boundary is sufficiently smooth, a satisfactory condition can be formulated and is so formulated in the present paper. This result is sufficiently general so as to yield the analogous results given by Aronszajn and Schechter for partial differential equations. When the results here given are applied to the theory of partial differential equations, one obtains necessary and sufficient conditions for Gaarding's inequality to hold.

In a certain sense the present paper is incomplete in that the implications of index theory to multiple integral problems have not been carried out and the applications to boundary value problems have been omitted. The extension of the theory of conjugate points and focal points to multiple integral problems has been omitted also. This extension has been carried out in part by Dennemeyer [6].

As is to be expected, the theory developed here is applicable to the theory of partial differential equations of elliptic type. These applications are developed in part in $\S \S 12$ and 13 , where it is recalled again that conditions of ellipticity for differential equations are equivalent to conditions of Legendre of certain integrals. It is shown, in particular, that if a partial differential equation is elliptic in a suitable sense, the strong solutions always exist for the compatible case. This is done without first establishing the existence of weak solutions. Moreover, differentiability theorems are obtained. Again the discussion is incomplete. However, it is hoped that sufficient material has been given to illustrate the applications to partial differential equations.

The literature on this subject is large. A brief list of references is given at the end of the paper. Further references can be found in these papers. As is to be expected, there is a close connection between the ideas and methods presented in the present paper and in the earlier paper to those given by various writers in the theory of partial differential equations. In this connection the 
author is particularly indebted to Aronszajn, Browder, Friedrichs, Gaarding, Hörmander, Morrey, Nirenberg, Schechter and van Hove. In particular free use has been made of the ideas given by Schechter.

The results here given can be used to establish a very general sufficiency theorem for weak relative minima for multiple integral problems in the calculus of variations. The proof can be made by the indirect method used previously by the author [12].

2. Preliminary remarks. The purpose of this section is to recall some results in the theory of Hilbert space that will be useful in the present paper. We shall use the notations and terminology used previously by the author [11], except for some minor exceptions.

Let $\mathfrak{A}$ be a Hilbert space over the field of reals or over the field of complexes. The symbols $x, y, z, \cdots$ normally will denote elements of $\mathscr{A}$ and the symbols $a, b, c, \cdots$ will denote scalars. As is customary, we denote the inner product by $(x, y)$ and the norm by $\|x\|=(x, x)^{1 / 2}$. A sequence $\left\{x_{q}\right\}$ in $\mathfrak{A}$ will be said to converge weakly to $x_{0}$, written $x_{q} \rightarrow x_{0}$, if $\left(x_{q}, y\right) \rightarrow\left(x_{0}, y\right)$ for each $y$ in $\mathfrak{A}$. A sequence $\left\{x_{q}\right\}$ in $\mathfrak{A}$ will be said to converge strongly to $x_{0}$, written $x_{q} \Rightarrow x_{0}$, if $\left\|x_{q}-x_{0}\right\| \rightarrow 0$.

A bounded linear functional $L(x)$ on $\mathfrak{A}$ will be called a linear form on $\mathfrak{A}$. A functional $Q(x, y)$ on $\mathfrak{A} \times \mathfrak{A}$ will be called a bilinear form in $\mathfrak{A}$ if $Q(x, y)$ and the conjugate $\bar{Q}(y, x)$ of $Q(y, x)$ are linear forms in $x$ for each $y$ in $\mathscr{A}$. If in addition $Q(x, y)=\bar{Q}(y, x)$, then $Q(x)=Q(x, x)$ will be called a quadratic form on $\mathfrak{A}$. The terms positive, nonnegative, negative, nonpositive quadratic forms on $\mathfrak{A}$ are self-explanatory. A quadratic form $Q(x)$ will be said to be positive definite on $\mathfrak{A}$ if there is a real number $h>0$ such that $Q(x) \geqq h\|x\|^{2}$ holds on $\mathfrak{A}$.

A quadratic form $K(x)$ will be said to be w-continuous (completely continuous) on $\mathscr{A}$ if $K\left(x_{q}\right) \rightarrow K\left(x_{0}\right)$ whenever $x_{q} \rightarrow x_{0}$. A quadratic form $Q(x)$ will be said to be wls-continuous on $\mathfrak{A}$ if $\lim _{\inf } \operatorname{s=\infty }_{q} Q\left(x_{q}\right) \geqq Q\left(x_{0}\right)$ whenever $x_{q} \rightarrow x_{0}$. A quadratic form $Q(x)$ is $w l s$-continuous if and only if it is expressible in the form

$$
Q(x)=P(x)+K(x)
$$

where $P$ is nonnegative and $K$ is $w$-continuous. If $Q$ is representable in the form (2.1) on $\mathfrak{A}$ as the sum of a positive definite form $P$ and a $w$-continuous form $K$, then $Q$ will be called a Legendre form. As was shown by the author a quadratic form $Q$ is a Legendre form if and only if it is $w l s$-continuous and $x_{q} \Rightarrow x_{0}$ whenever $x_{q} \rightarrow x_{0}$ and $Q\left(x_{q}\right) \rightarrow Q\left(x_{0}\right)$. Moreover it is a Legendre form if and only if it is positive definite in the orthogonal complement of a finite dimensional subspace of $\mathfrak{A}$. Legendre forms play a fundamental role in the calculus of variations. In the present paper they will be used in order to establish existence theorems for partial differential equations of elliptic type.

Given a quadratic form $J(x)$ we say that two vectors $x$ and $y$ are $J$-orthog- 
onal in case $J(x, y)=0$. The class of all vectors that are $J$-orthogonal to the vectors in a given class $B$ will be called the $J$-orthogonal complement of $B$ and will be denoted by $B^{J}$. Clearly $B^{J}$ is a subspace of $\mathfrak{A}$. The vectors in the class $B_{0}=B \cap B^{J}$ will be called the $J$-null vectors of $B$. The dimension of $B_{0}$ will be called the nullity of $J$ on $B$. If $B_{0}$ consists only of the vector $x=0$, then $J$ will be said to be nondegenerate on $B$. The quadratic form will be said to be nonsingular on a subspace $B$ of $\mathfrak{A}$ if given a linear form $L$ on $B$ there is a unique vector $y$ in $B$ such that $L(x)=J(x, y)$ on $B$. In this event $J$ is nondegenerate on $B$.

3. Auxiliary theorems. The purpose of this section is to establish certain properties of Legendre forms, that characterize these forms. These results are useful, not only in the existence theorems here given, but also in the theory of the second variation for simple and multiple integral problems in the calculus of variations.

Theorem 3.1. Let $P, Q$ be quadratic forms on $\mathfrak{A}$. If $P(x) \geqq Q(x)$ on $\mathfrak{A}$ and $Q$ is wls-continuous on $\mathfrak{A}$, then $P$ is wls-continuous on $\mathfrak{A}$. If $P(x) \geqq Q(x)$ on $\mathfrak{A}$ and $Q(x)$ is a Legendre form on $\mathfrak{A}$, then $P$ is a Legendre form on $\mathfrak{A}$. If $P$ is positive definite on $\mathfrak{A}$, then $Q$ is wlsc on $\mathfrak{A}$ if and only if for every positive number $\epsilon$ the quadratic form $Q+\epsilon P$ is a Legendre form on $\mathfrak{A}$. If $P$ is positive definite on $\mathfrak{A}$, then $Q$ is a Legendre form on $\mathfrak{A}$ if and only if there is a positive number $h$ such that $Q-h P$ is wls-continuous on $\mathfrak{A}$.

This result follows readily from the definitions and results given in the preceding section $[11$, pp. 545,553$]$.

TheOREM 3.2. Let $K$ be a w-continuous quadratic form on $\mathfrak{A}$ and let $J$ be a quadratic form on $\mathfrak{A}$. Suppose that $J(x)>0$ whenever $K(x)=0$ and $x \neq 0$. Then $J$ is a Legendre form on $\mathfrak{A}$ if and only if there is a real number $b$ such that $J+b K$ is positive definite on $\mathfrak{A}$.

This result has been established previously by the author $[11$, p. 561].

THEOREM 3.3. If $J$ is a quadratic form on $\mathfrak{A}$ then there exist unique subspaces $\mathfrak{A}_{+}, \mathfrak{P}_{0}, \mathfrak{A}_{-}$of $\mathfrak{A}$ that are mutually orthogonal and $J$-orthogonal and are such that $J$ is positive on $\mathfrak{A}_{+}$, is negative on $\mathfrak{A}_{-}$and is zero on $\mathfrak{A}_{0}$. The class $\mathfrak{A}_{0}$ is the class of $J$-null vectors on $\mathfrak{A}$.

This result follows from a standard theorem on bounded linear operators and has been established by the author $[11$, p. 543].

CoROllaRY 1. If the dimension of $\mathfrak{A}_{0}$ is infinite or if the dimensions of $\mathfrak{A}_{+}$ and $\mathfrak{A}_{-}$are both infinite there exists an infinite dimensional subspace $\mathfrak{e}$ of $\mathfrak{A}$ on which $J$ is w-continuous.

If $\mathfrak{A}_{0}$ is of infinite dimension, select $\mathfrak{C}=\mathfrak{A}$. Consider next the case in which $\mathfrak{A}_{+}$and $\mathfrak{P}_{-}$are of infinite dimension. Let $\left\{y_{n}\right\}$ and $\left\{z_{n}\right\}$ be orthonormal se- 
quences in $\mathfrak{A}_{+}$and $\mathfrak{A}_{-}$respectively and having the additional property that $J\left(y_{m}, y_{n}\right)=J\left(z_{m}, z_{n}\right)=0(m \neq n)$. Set $x_{n}=y_{n} \cos \theta_{n}+z_{n} \sin \theta_{n}$, where $\theta_{n}$ is chosen so that

$$
\text { . } J\left(x_{n}\right)=J\left(y_{n}\right) \cos ^{2} \theta_{n}+J\left(z_{n}\right) \sin ^{2} \theta_{n}=0, \quad n=1,2,3, \cdots .
$$

Since $J\left(x_{m}, x_{n}\right)=0$, as one readily verifies, it follows that $J(x) \equiv 0$ on the subspace $\mathcal{C}$ of $\mathfrak{A}$ spanned by the orthonormal sequence $\left\{x_{n}\right\}$. Hence $J$ is $w$ continuous on $\mathfrak{C}$, as was to be proved.

Observe that if we choose $\theta_{n}$ above so that $0<J\left(x_{n}\right)<1 / 2^{n}$ then $J$ will be positive on $\mathfrak{C}$, as well as $w$-continuous on $\mathcal{C}$. In this selection it is immaterial whether $z_{n}$ is in $\mathfrak{A}_{-}$or in $\mathfrak{A}_{0}$. Hence we have

CoRollary 2. If $\mathfrak{Q}_{+}$is of infinite dimension and if at least one of the subspaces $\mathfrak{A}_{0}$ and $\mathfrak{A}_{-}$is of infinite dimension, then there is an infinite dimensional subspace $\mathfrak{e}$ on which $J$ is positive and w-continuous.

As a further result in this direction we have

Theorem 3.4. A positive quadratic form $P$ on $\mathfrak{A}$ is positive definite on $\mathfrak{A}$ if and only if every subspace $\mathfrak{C}$ of $\mathfrak{A}$ on which $P$ is w-continuous is of finite dimension. A positive quadratic form is positive definite on $\mathfrak{A}$ if and only if for every linear form $L(x)$ there is a vector $y$ in $\mathfrak{A}$ such that $L(x)=P(x, y)$ for all $x$ in $\mathfrak{A}$.

Suppose that $P$ is $w$-continuous on a subspace $\mathcal{C}$ of $\mathfrak{A}$ and that $P$ is positive definite on $\mathfrak{A}$. Let $\left\{x_{n}\right\}$ be a sequence in $\mathfrak{C}$ that converges weakly to a vector $x_{0}$. Then $P\left(x_{n}\right) \rightarrow P\left(x_{0}\right)$ and hence $x_{n} \Rightarrow x_{0}$. Hence weak and strong convergence are equivalent on $\mathfrak{C}$ and $\mathfrak{C}$ must be of finite dimension.

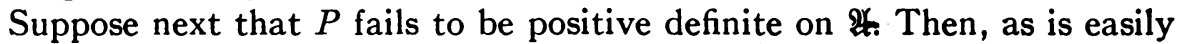
seen, one can select an orthonormal sequence $\left\{x_{n}\right\}$ such that $J\left(x_{m}, x_{n}\right)$ $=0(m \neq n)$ and $J\left(x_{n}\right) \leqq 1 / 2^{n}$. Then, on the space spanned by $\left\{x_{n}\right\}$ we have

$$
J(x)=\sum_{n=1}^{\infty} J\left(x_{n}\right)\left|a_{n}\right|^{2}
$$

where $a_{n}=\left(x, x_{n}\right)$. Since $J\left(x_{n}\right) \leqq 1 / 2^{n}$ it follows that $J(x)$ is $w$-continuous on e. The last statement was established previously. This proves the theorem.

Lemma 3.5. Let $J$ be a quadratic form on $\mathfrak{2}$. Then either $J$ or $-J$ is a Legendre form on $\mathfrak{A}$ if and only if $x_{n} \Rightarrow 0$ whenever $x_{n} \rightarrow 0, J\left(x_{n}\right) \rightarrow 0$. Moreover, $J$ or $-J$ is a Legendre form on $\mathfrak{A}$ if and only if every subspace $\mathfrak{C}$ of $\mathfrak{A}$ on which $J$ is w-continuous is of finite dimension.

The first statement in the theorem has been established previously by the author [11, p. 553]. In order to prove the second statement suppose that no subspace $\mathfrak{C}$ of $\mathfrak{A}$ on which $J$ is $w$-continuous is of infinite dimension. Then, by Corollary 1 , the dimension of $\mathfrak{A}_{0}$ is finite, as is the dimension of $\mathfrak{A}_{+}$or of 


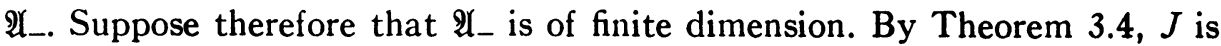
positive definite on $\mathfrak{A}_{+}$. Since $\mathfrak{A}_{+}$is the orthogonal complement of a subspace, namely $\mathfrak{A}_{0}+\mathfrak{A}_{-}$, of finite dimension, it follows that $J$ is a Legendre form. The converse follows readily from the first statement in the theorem.

CoRollary. If $J$ is a Legendre form on $\mathfrak{A}$, the subspaces $\mathfrak{A}_{0}$ and $\mathfrak{A}_{-}$described in Theorem 3.3 are of finite dimension. In particular $J$ is of finite nullity on $\mathfrak{A}$.

This result follows from the fact that $-J(x) \geqq 0$ on $\mathfrak{C}=\mathfrak{P}_{0}+\mathfrak{A}_{-}$and hence is $w l s$-continuous on $\mathfrak{C}$. Since $J$ is also $w l s$-continuous on $\mathfrak{e}$, it follows that $J$ is $w$-continuous on $\mathcal{C}$. Hence $\mathcal{C}$ is of finite dimension, as was to be proved. The nullity of $J$ on $\mathfrak{A}$ is the dimension of $\mathfrak{A}_{0}$.

ThEOREM 3.6. Let $J(x)$ be a quadratic form on $\mathscr{A}$ and let $B$ be a subspace of $\mathfrak{A}$. Suppose that $\mathbb{B}$ possesses an infinite dimensional subspace on which $J$ is nondegenerate, then either $J$ or $-J$ is a Legendre form on $B$ if and only if one of the following conditions is met.

(a) The relation $\mathfrak{A}=\mathfrak{C}+\mathfrak{C}^{J}$ holds for every subspace $\mathfrak{C}$ of $\mathbb{B}$ on which $J$ is nondegenerate.

(b) The relation $\left(\mathfrak{C} \cap \mathfrak{C}^{J}\right)^{J}=\mathfrak{C}+\mathfrak{C}^{J}$ holds for every subspace $\mathfrak{C}$ of $\mathbb{B}$.

(c) Given a subspace $\mathcal{C}$ of $B$ on which $J$ is nondegenerate and a linear form $L$, there is a unique vector $y$ in $\mathfrak{e}$ such that $L(x)=J(x, y)$ for all $x$ in $\mathfrak{C}$.

(d) Given a subspace $\mathcal{C}$ of $B$ and a linear form $L$ such that $L(x)=0$ on $\mathfrak{e} \cap \mathfrak{e}^{J}$, then there is a vector $y$ in $\mathfrak{C}$ such that $L(x)=J(x, y)$ for all $x$ in $\mathfrak{C}$.

The equivalence of the conditions (a), (b), (c), (d) has been established previously by the author $\left({ }^{3}\right)$. Moreover, it was shown that these conditions are all necessary.

Suppose now that condition (c) holds. Then by Theorem $3.4 J$ is positive definite on every subspace $B_{+}$of $B$ on which it is positive and negative definite on every subspace $B_{-}$of $B$ on which it is negative. This is possible, by Theorem 3.4 and the corollaries to Theorem 3.3, if and only if $J$ is $w$-continuous on no infinitely dimensional subspace $\mathcal{C}$ of $\mathbb{B}$. Hence either $J$ or $-J$ is a Legendre form on $B$, by virtue of the last theorem.

THEOREM 3.7. Let $J$ be a Legendre form and let $K(x)$ be a positive w-continuous quadratic form. A quadratic form $H(x)$ is w-continuous on $\mathfrak{A}$ if and only if given an $\epsilon>0$ there is a constant $b$ such that

$$
|H(x)| \leqq \epsilon J(x)+b K(x)
$$

holds on $\mathfrak{A}$.

(3) $[11, \mathrm{pp} .554-556]$. In this reference, these conditions were given necessary and sufficient conditions for "quasinonsingularity," and it was shown that every Legendre form was quasinonsingular. The converse was not estahlished. In Theorem 12.4 of the paper cited one should add "and of finite nullity." 
If $H(x)$ is $w$-continuous, then $\epsilon J+H$ and $\epsilon J-H$ are Legendre forms, where $\epsilon>0$. By virtue of Theorem 3.2 there is a constant $b>0$ such that $\epsilon J+H+b K$ and $\epsilon J-H+b K$ are positive definite. Hence (3.1) holds. Conversely, suppose that for every positive number $\epsilon$ there is a positive number $b$ such that (3.1) holds. If $\left\{x_{n}\right\}$ is a sequence in $\mathscr{P}$ such that $x_{n} \rightarrow 0$, it follows from (3.1) that

$$
\limsup _{n=\infty}\left|H\left(x_{n}\right)\right| \leqq \epsilon \limsup _{n=\infty} J\left(x_{n}\right) .
$$

Since $\epsilon$ is arbitrary we have $\lim _{n=\infty} H\left(x_{n}\right)=0$, that is, $H$ is $w$-continuous, as was to be proved.

4. Connections between Legendre forms and operators. Consider now a second Hilbert space $\mathfrak{A}^{\prime}$ having the same scalars as $\mathfrak{A}$. Its elements will be denoted by $x^{\prime}, y^{\prime}, \ldots$, its inner product by $\left(x^{\prime}, y^{\prime}\right)^{\prime}$ and its norm by $\left\|x^{\prime}\right\|^{\prime}$.

Let $A$ be a bounded linear operator on $\mathfrak{A}$ to $\mathfrak{A}^{\prime}$. Let $\mathfrak{Q}_{A}$ be the range of $A$ and $\mathfrak{N}_{A}$ its null space, that is, all $x$ in $\mathfrak{A}$ such that $A x=0$. The dimension of $\mathfrak{N}_{A}$ will be called the nullity of $A$ on $\mathfrak{\Re}$. We shall be concerned with finding conditions which will insure the solvability of the equation $A x=x^{\prime}$, when $x^{\prime}$ is prescribed. Clearly if a solution exists, then $x^{\prime}$ must be orthogonal to the null space of the adjoint $A^{*}$ of $A$. This condition is sufficient if and only if the range $R_{A}$ of $A$ is the orthogonal complement of the null space of $A^{*}$, that is, if and only if $R_{A}$ is closed. A criterion that $R_{A}$ be closed is given in the following.

THEOREM 4.1. A necessary and sufficient condition that the operator $A$ be of finite nullity and that its range be closed is that the quadratic form $J(x)=J(x, x)$ defined by the bilinear form

$$
J(x, y)=(A x, A y)^{\prime}
$$

be a Legendre form on $\mathfrak{A}$.

Observe that since $J(x)=\|A x\|^{\prime 2}=0$ if and only if $A x=0$ the $J$-orthogonal complement $\mathfrak{Q}^{J}$ of $\mathfrak{A}$ is $\mathfrak{N}_{A}$. Suppose now that $J$ is a Legendre form on $\mathfrak{Q}$. Then the dimension of $\mathfrak{N}_{A}=\mathfrak{A}_{0}=\mathfrak{Q P}^{J}$ is finite, by the corollary to Theorem 3.6. Let $y^{\prime}$ be an element in the closure of $\mathbb{R}_{A}$. The linear form

$$
L(x)=J\left(A x, y^{\prime}\right)
$$

vanishes on $\mathfrak{R}_{\boldsymbol{A}}$. By Theorem 3.6 there is accordingly a vector $y$ in $\mathfrak{A}$ such that the relation

$$
0=L(x)-J(x, y)=\left(A x, y^{\prime}-A y\right)
$$

holds for all $x$ in $\mathfrak{A}$. Since $y^{\prime}$ is in the closure of $\mathfrak{R}_{A}$ this is possible only in case $y^{\prime}=A y$. Hence $R_{A}$ is closed.

Suppose next that $A$ is of finite nullity and that its range is closed. Then 
$J$ is of finite nullity. We can assume that $\mathfrak{R}_{A}=0$ since this can be obtained by restricting $A$ to the orthogonal complement of $\mathfrak{R}_{A}$. Moreover we can suppose that $\mathfrak{U}^{\prime}=\mathfrak{R}_{A}$. Then $A$ has an inverse $A^{-1}$ whose domain is in $\mathfrak{U}^{\prime}$. Since $A$ is closed so also is $A^{-1}$. It follows that $A^{-1}$ is bounded. There is accordingly a constant $m>0$ such that

$$
\left\|A^{-1} x^{\prime}\right\| \leqq \frac{1}{m}\|x\|^{\prime} .
$$

Setting $x=A^{-1} x^{\prime}$, i.e., $x^{\prime}=A x$ we have

$$
J(x)=\|A x\|^{\prime 2} \geqq m^{2}\|x\|^{2} .
$$

Consequently $J$ is positive definite and hence is a Legendre form, as was to be proved.

CoRollary. The range of $A$ is closed if and only if the quadratic form $J$ defined by (4.1) is positive definite on the orthogonal complement of its null space $\mathfrak{N}_{A}$.

As a further result we have

THEOREM 4.2. If $\mathfrak{A}^{\prime}=\mathfrak{i}$ and the quadratic form $J_{1}(x)=J_{1}(x, x)$ defined by the bilinear form

$$
J_{1}(x, y)=(A x, y)+(x, A y)
$$

is a Legendre form on $\mathfrak{A}$, then $A$ is of finite nullity and the range of $A$ is closed.

It is sufficient, by Theorem 4.1, to show that the quadratic form $J$ defined by (4.1) is also a Legendre form. Suppose that this is not the case. Then, by Theorem 3.5, there is an infinite dimensional subspace $\mathfrak{C}$ of $A$ on which $J$ is $w$-continuous. Let $\left\{x_{q}\right\}$ be a sequence in $\mathfrak{e}$ such that $x_{q} \rightarrow 0$. Then $J\left(x_{q}\right) \rightarrow 0$, that is, $A x_{q} \Rightarrow 0$. Consequently, $\left(x_{q}, A x_{q}\right) \rightarrow 0$ and hence $J_{1}\left(x_{q}\right) \rightarrow 0$. The quadratic form $J_{1}$ is therefore w-continuous on $\mathcal{C}$. This is impossible, by Theorem 3.5, since $J_{1}$ is Legendre form. This proves the theorem.

Corollary. If $\mathfrak{H}^{\prime}=\mathfrak{A}$ and $J_{1}$ is a Legendre form on the orthogonal complement of $\mathfrak{R}_{A}$, then the range of $A$ is closed.

Theorem 4.3. Suppose that the nullity of $A$ is finite and let $C$ be a compact linear operator on $\mathfrak{A}$ to a Hilbert space $\mathfrak{A}^{\prime \prime}$. Let $B$ be the operator on $\mathfrak{A}$ to the cartesian product $\mathfrak{A}^{\prime} \times \mathfrak{H}^{\prime \prime}$ defined by the pair $\{A x, C x\}$. The range of $A$ is closed if and only if the range of $B$ is closed. If $\mathfrak{A}^{\prime \prime}=\mathfrak{H}^{\prime}$, then the range of $A$ is closed if and only if the range of $B=A+C$ is closed. In either event if the range of $A$ is closed, the nullity of $B$ is finite.

This result is obtained by applying Theorem 4.1. In the first instance the range of $B$ is closed if and only if the quadratic form $J_{2}$ defined by 


$$
J_{2}(x)=J(x)+K(x), \quad J(x)=\|A x\|^{\prime 2}, \quad K(x)=\|C x\|^{\prime \prime 2}
$$

is a Legendre form on $\mathfrak{A}$. Since $C$ is compact, $K(x)$ is $w$-continuous. Consequently $J_{2}$ is a Legendre form if and only if $J$ is a Legendre form. It follows that the first conclusion in the theorem holds.

Suppose next that $\mathfrak{Q}^{\prime \prime}=\mathfrak{A}^{\prime}$ and $B=A+C$. Then

$$
K(x)=(A x, C x)^{\prime}+(C x, A x)^{\prime}+(C x, C x)^{\prime}
$$

is $w$-continuous on $\mathfrak{A}$. Hence $J$ is a Legendre form on $\mathfrak{A}$ if and only if

$$
\|B x\|^{\prime 2}=J(x)+K(x)
$$

is a Legendre form on $\mathfrak{A}$. This proves the theorem.

5. Some theorems on integral means and related results. Before studying applications of the results given in the preceding pages, it will be convenient to have at hand certain properties of a type of integral mean that will be useful in the pages that follow. To this end let $T$ be an interval $a \leqq t \leqq b$ in an $m$-dimensional euclidean space of points $t=\left(t_{1}, \cdots, t_{m}\right)$. Let $x(t)$ be an integrable function on $T$ and set

$$
x^{h}(t)=\frac{1}{|T|} \int_{T} x(t+h(s-t)) d s, \quad 0<h \leqq 1,
$$

where $|T|$ denotes the measure of $T$. For each $h$ on $0<h \leqq 1$ the function $x^{h}$ will be called the $h$-average of $T$. This integral mean retains the essential properties of the integral means normally used and has the advantage that it is defined over the whole region $T$ without extending the function to be defined on the complement of $T$. This property is particularly useful when derivatives of the function are involved.

The properties of this function will be stated in a sequence of lemmas. In describing these properties we shall sometimes center our attention on a particular component $t_{\sigma}$ of $t$; we denote the set of complementary components by $t_{\sigma}^{\prime}$ and shall write $t=\left(t_{\sigma}, t_{\sigma}^{\prime}\right)$. Thus the interval $a \leqq t \leqq b$ is the cartesian product of $a_{\sigma} \leqq t_{\sigma} \leqq b_{\sigma}$ and $a_{\sigma}^{\prime} \leqq t_{\sigma}^{\prime} \leqq b_{\sigma}^{\prime}$. Occasionally we shall use the abbreviations:

$$
\begin{array}{lll}
\rho=t+h(s-t), & \rho_{\sigma}=t_{\sigma}+h\left(s_{\sigma}-t_{\sigma}\right), & \rho_{\sigma}^{\prime}=t_{\sigma}^{\prime}+h\left(s_{\sigma}^{\prime}-t_{\sigma}^{\prime}\right), \\
\tau=(1-h) t, & \tau_{\sigma}=(1-h) t_{\sigma}, & \tau_{\sigma}^{\prime}=(1-h) t_{\sigma}^{\prime} .
\end{array}
$$

In our proof we shall assume that $a_{\sigma}=0, b_{\sigma}=1$. Then $x^{h}$ is given in the simpler forms

$$
x^{h}(t)=\int_{0}^{1} x(\rho) d s=\frac{1}{h^{m}} \int_{\tau}^{\tau+h} x(\rho) d \rho=\frac{1}{h} \int_{\tau_{\sigma}}^{\tau_{\sigma}+h} \int_{0}^{1} x\left(\rho_{\sigma}, \rho_{\sigma}^{\prime}\right) d \rho_{\sigma} d s_{\sigma}^{\prime} .
$$

With these notations in mind we can prove a sequence of lemmas. In these 
lemmas it is understood that $x$ is a real or complex integrable function of $t$ on $T$.

LEMMA 5.1. The h-average $x^{h}$ of $x$ is a continuous function on $T$ for each $h$ on $0<h \leqq 1$ and $\lim _{h=0} x^{h}(t)=x(t)$ almost everywhere on $T$. If $x$ is in $\mathfrak{L}_{p}(p \geqq 1)$, there is a constant $M$ such that

$$
\int_{T}\left|x^{h}(t)\right|^{p} \leqq M \int_{T}|x(t)|^{p} d t .
$$

Moreover

$$
\lim _{h=0} \int_{T}\left|x^{h}(t)-x(t)\right| p d t=0 .
$$

If $\left\{x_{q}\right\}$ is a sequence of functions in $\mathfrak{S}_{p}$ converging weakly to $x$, then for each $h$ on $0<h \leqq 1$ one has $\lim _{q-\infty} x_{q}^{h}(t)=x^{h}(t)$ uniformly on $T$.

These results can be established by an argument like those in proving the analogous results for standard integral means. The same is true for the results given in the following lemma. In the proof of the lemma one uses the formula

$$
\frac{\partial x^{h}}{\partial t_{\sigma}}=\frac{1-h}{h} \int_{0}^{1}\left[x\left(\tau_{\sigma}+h, \rho_{\sigma}^{\prime}\right)-x\left(\tau_{\sigma}, \rho_{\sigma}^{\prime}\right)\right] d s_{\sigma}^{\prime}
$$

which holds almost everywhere on $T$.

LEMMA 5.2. For each integer $\sigma \leqq m$ the function $x^{h}$ is absolutely continuous in $t_{\sigma}$ on $a_{\sigma} \leqq t_{\sigma} \leqq b_{\sigma}$ for each $t_{\sigma}^{\prime}$ on $a_{\sigma}^{\prime} \leqq t_{\sigma}^{\prime} \leqq b_{\sigma}^{\prime}$. If $x$ is in $\mathfrak{L}_{p}(p \geqq 1)$ so also is $\partial x^{h} / \partial t_{\sigma}$. Moreover, there is a constant $N$ independent of $h$ such that

$$
\int_{T}\left|\frac{\partial x^{h}}{\partial t_{\sigma}}\right|^{p} d t \leqq \frac{N}{h^{p}} \int_{T}|x(t)| p d t .
$$

If for almost all $t_{\sigma}^{\prime}$ on $a_{\sigma}^{\prime} \leqq t_{\sigma}^{\prime} \leqq b_{\sigma}^{\prime}$, the function $x$ is absolutely continuous in $t_{\sigma}$ on $a_{\sigma} \leqq t_{\sigma} \leqq b$ and $\partial x / \partial t_{\sigma}$ is integrable on $T$, then

$$
\frac{\partial x^{h}}{\partial t_{\sigma}}=(1-h)\left(\frac{\partial x}{\partial t_{\sigma}}\right)^{h} .
$$

The following lemma will be useful. By the support set of a function $x(t)$ will be meant the closure of the set of points at which $x(t) \neq 0$.

Lemma 5.3. Suppose that $\lim _{h=0} x^{h}(t)=x(t)$ for each $t$ in $T$ for which the limit $\lim _{h=0} x^{h}(t)$ exists. Then given an integer $\sigma \leqq n$ the following statements are equivalent:

(1) There exists an integrable function $x_{\sigma}(t)$ on $T$ such that 


$$
\int_{T}\left\{x(t) \frac{\partial y(t)}{\partial t_{\sigma}}+x_{\sigma}(t) y(t)\right\} d t=0
$$

for every function of class $C^{\infty}$ whose support set is interior to $T$.

(2) There exists an integrable function $x_{\sigma}(t)$ on $T$ such that

$$
\int_{c_{\sigma}}^{d_{\sigma}^{\prime}}\left\{x\left(d_{\sigma}, t_{0}^{\prime}\right)-x\left(c_{\sigma}, t_{\sigma}^{\prime}\right)\right\} d t_{\sigma}^{\prime}=\int_{0}^{d} x_{\sigma}(t) d t_{\sigma}
$$

holds for all intervals $c \leqq t \leqq d$ in $T$ except for $(c, d)$ on a set of $2 m$-dimensional measure zero.

(3) The function $x(t)$ is absolutely continuous in $t_{\sigma}$ on $a_{\sigma} \leqq t_{\sigma} \leqq b_{\sigma}$ for almost all $t_{\sigma}^{\prime}$ on $a_{\sigma}^{\prime} \leqq t_{\sigma}^{\prime} \leqq b_{\sigma}^{\prime}$ and $\partial x / \partial t_{\sigma}$ is integrable on $T$.

If $x$ has these properties, then $x_{\sigma}=\partial x / \partial t_{\sigma}$ almost everywhere on $T$ and the formula (5.8) holds for every interval $c \leqq t \leqq d$ in $T$.

This result has been established by Calkin [5] and Morrey $[15 ; 16]$.

Corollary. Let $\left\{x_{q}\right\}$ be a sequence of functions of class $C^{\prime}$ on $T$ converging in the mean of order $p$ to a function $x$ on $T$, where $x$ has been normalized so that $\lim x^{h}(t)=x(t)$ whenever $\lim _{h=0} x^{h}(t)$ exists. If the sequence of derivatives $\left\{\partial x_{q} / \partial t_{\sigma}\right\}$ converges in the mean of order $p$ on $T$ to a function $x_{\sigma}$, then $x$ and $x_{\sigma}$ are related as described in Theorem 5.3.

This result follows from the fact that the relation (5.7) holds with $x, x_{\sigma}$ replaced by $x_{q}$ and $\partial x_{q} / \partial t_{\sigma}$ and hence also for the functions $x, x_{\sigma}$ given in the corollary.

LEMma 5.4. Suppose that, for each integer $\sigma \leqq m, x$ is absolutely continuous in $t_{\sigma}$ on $a_{\sigma} \leqq t_{\sigma} \leqq b_{\sigma}$ for almost all $t_{\sigma}^{\prime}$ on $a_{\sigma}^{\prime} \leqq t_{\sigma}^{\prime} \leqq b_{\sigma}^{\prime}$. Suppose further that the functions $x$ and $x_{\sigma}=\partial x / \partial t_{\sigma}(\sigma=1, \cdots, m)$ are in $\mathfrak{L}_{p}(p \geqq 1)$. There is a constant $M$ independent of $h$ such that

$$
\int_{T}\left|x^{h}(t)-x(t)\right|^{p} d t \leqq h^{p} M \int_{T}\left[\left|x_{1}\right|^{2}+\cdots+\left|x_{m}\right|^{2}\right]^{p / 2} d t .
$$

In view of the last corollary it is sufficient to prove the theorem under the hypothesis that $x(t)$ is of class $C^{\prime}$ on $T$. Consider the difference

$$
x^{h}(t)-x(t)=\int_{0}^{1}[x(\rho)-x(t)] d s=h \int_{0}^{1} f(t, \theta h) d \theta
$$

where

$$
\left.f(t, h)=\int_{0}^{1}\left(s_{\sigma}-t_{\sigma}\right) x_{\sigma}(\rho) d s \quad \text { ( } \quad \text { summed }\right) .
$$

An application of Hölder's inequality yields 


$$
|f(t, h)|^{p} \leqq \int_{0}^{1}\left[x_{\sigma}(\rho) \bar{x}_{\sigma}(\rho)\right]^{p / 2} d s
$$

Hence there is a constant $M$ such that

$$
\int_{0}^{1}|f(t, h)|^{p} d t \leqq \int_{0}^{1} \int_{0}^{1}\left[x_{\sigma}(\rho) \bar{x}_{\sigma}(\rho)\right]^{p / 2} d t d s \leqq M \int_{0}^{1}\left[x_{\sigma} \bar{x}_{\theta}\right]^{p / 2} d t .
$$

Combining this with (5.10) it follows that (5.9) holds.

Lemma 5.5. Let $v(t)$ be Lipschitzian on $T$ and set

$$
z^{h}(t)=\frac{1}{|T|} \int_{T}[v(\rho)-v(t)] x(\rho) d s=(v x)^{h}-v x^{h} .
$$

If $x$ is in $\mathfrak{L}_{p}(p \geqq 1)$ then

$$
\lim _{h=0} \int_{T}\left|z^{h}(t)\right|^{p} d t=0, \quad \lim _{h=0} \int_{T}\left|\frac{\partial z^{h}}{\partial t_{\sigma}}\right|^{p} d t=0 \quad(\sigma=1, \cdots, m) .
$$

This result has been established by Friedrichs for mollifiers [7]. It holds for all standard forms of the integral mean. The first limit in (5.2) follows from Lemma 5.1 and the definition of $z^{h}$. As a first step in the proof of the second limit in (5.12) we shall show that there is a constant $N$ independent of $h$ such that

$$
\int_{0}^{1}\left|\frac{\partial z^{h}}{\partial t_{\sigma}}\right|^{p} d t \leqq N^{p} \int_{0}^{1}|x(t)|^{p} d t
$$

To this end we write

$$
\frac{2^{h}\left(t_{\sigma}+r, t_{\sigma}^{\prime}\right)-z^{h}(t)}{r}=A_{1}+A_{2}-A_{8}
$$

where

$$
\begin{aligned}
A_{1} & = \pm \frac{1}{r h} \int_{\tau_{0}}^{\mu_{0}} B d s_{\sigma}, \quad \mu_{0}=\tau_{\sigma}+(1-h) r, \\
A_{2} & = \pm \frac{1}{r h} \int_{h+\tau_{\sigma}}^{h+\mu_{\sigma}} B d s_{\sigma}, \\
B & =\int_{0}^{1}\left[v\left(s_{\sigma}, \rho_{\sigma}^{\prime}\right)-v(t)\right] x\left(s_{\sigma}, \rho_{\sigma}^{\prime}\right) d s_{\sigma}^{\prime}, \\
A_{3} & =\frac{v\left(t_{\sigma}+r\right)-v(t)}{r} x^{h}(t) .
\end{aligned}
$$


Since $v(t)$ is Lipschitzian we may select a constant $k$ such that

$$
|v(s)-v(t)| \leqq k|s-t| \text {. }
$$

Since $|s-t|^{2} \leqq m h^{2}$ we have

$$
\left|A_{1}\right| \leqq \frac{k m^{1 / 2}}{r} \int_{\tau_{\sigma}}^{\mu_{\sigma}} \int_{0}^{1}\left|x\left(s_{\sigma}, \rho_{\sigma}^{\prime}\right)\right| d s_{\sigma} d s_{\sigma}^{\prime} .
$$

From this result it follows that there is a constant $L$ such that

$$
\int_{0}^{1}\left|A_{1}\right|^{p} d t \leqq K^{p} m^{p / 2} L \int_{0}^{1}|x(t)|^{p} d t
$$

This formula also holds with $A_{1}$ replaced by $A_{2}$. Finally

$$
\int_{0}^{1}\left|A_{3}\right|^{n} d t \leqq N^{p} \int_{0}^{1}\left|x^{h}(t)\right|^{p} d t \leqq N^{p} M \int_{0}^{1}|x(t)|^{p} d t .
$$

The inequality (5.13) follows from these inequalities.

In order to prove the second limit in (5.12), observe that it holds if $x$ is of class $C^{\prime}$, as can be seen by differentiation. Given $x$ in $\mathscr{L}_{p}$ choose a sequence of functions $\left\{x_{q}\right\}$ of class $C^{\prime}$ converging in the mean of order $p$ to $x$. Writing

$$
\|x\|=\left[\int_{0}^{1}|x(t)| p d t\right]^{1 / p}
$$

and letting $z_{q}^{h}$ be defined by (5.11) with $x$ replaced by $x_{q}$ we have

$$
\left\|\frac{\partial z_{q}^{h}}{\partial t_{\sigma}}-\frac{\partial z^{h}}{\partial t_{\sigma}}\right\| \leqq N\left\|x_{q}-x\right\|
$$

by (5.13). Consequently

$$
\left\|\frac{\partial z^{h}}{\partial t_{\sigma}}\right\| \leqq\left\|\frac{\partial z_{q}^{h}}{\partial t_{\sigma}}\right\|+\left\|\frac{\partial z_{q}^{h}}{\partial t_{\sigma}}-\frac{\partial z^{h}}{\partial t_{\sigma}}\right\| \leqq\left\|\frac{\partial z_{q}^{h}}{\partial t_{\sigma}}\right\|+N\left\|x_{q}-x\right\| .
$$

Hence

$$
\limsup _{h=0}\left\|\frac{\partial z^{h}}{\partial t_{\sigma}}\right\| \leqq N\left\|x_{q}-x\right\| .
$$

Since $\left\|x_{q}-x\right\| \rightarrow 0$ it follows that $\lim _{h=0}\left\|\partial z^{h} / \partial t_{\sigma}\right\|=0$, as was to be proved.

6. Hilbert space $\mathfrak{H C}_{k}$ and $\mathcal{H}$. A real or complex valued function $x(t)$ will be said to be of class $D^{0}$ on an interval $T$ in an $m$-dimensional space of points $t=\left(t_{1}, \cdots, t_{m}\right)$ if it is integrable on $T$, if $x(t)$ is equal to the $\operatorname{limit}_{\lim } \lim _{h=0} x^{h}(t)$ of its $h$-average $x^{h}$ whenever this limit exists and if $x(t)=0$ elsewhere. It will 
be said to be of class $D^{(1)}$ on $T$ if it is of class $D^{0}$ and if there exist functions $x_{1}, \cdots, x_{m}$ of class $D^{0}$ on $T$ such that (5.7) holds for every interval $\mathrm{c} \leqq t \leqq d$ in $T$. In view of Lemma 5.3 the function $x_{\sigma}(t)$ will be called the partial derivative $\left(\partial x / \partial t_{\sigma}\right)$ of $x$ on $T$. A function $x$ on $T$ will be of class $D^{(k)}(k>1)$ on $T$ if it is of class $D^{(k-1)}$ and its $(k-1)$ st partial derivatives are of class $D^{(1)}$. Finally, a function will be said to be of class $D^{(k)}$ on an open set $T$ if it is of class $D^{(k)}$ on every interval in $T$. It should be observed that if $x$ is of class $D^{(k)}$ on $T$ and its $k$ th derivatives are bounded then $x$ is of class $C^{k-1}$ and its $(k-1)$ st derivatives are Lipschitzian. In this event $x$ will be said to be of class $B^{(k)}$ on $T$.

Let $\mathcal{H}_{k}$ be the class of all complex valued functions $x^{j}(t)(j=1, \cdots, n)$ of class $D^{k}$ on an open set $T$ which together with its partial derivatives of orders $\leqq k$ are square integrable on $T$. Given a set of $m$ nonnegative integers $\alpha=\left(\alpha_{1}, \cdots, \alpha_{m}\right)$ let

$$
x_{\alpha}^{j}=(-i)^{|\alpha|} \frac{\partial^{|\alpha|} x^{j}}{\partial t_{1}^{\alpha_{1}} \cdots \partial t_{m}^{\alpha_{m}}} \quad|\alpha|=\alpha_{1}+\cdots+\alpha_{m} .
$$

If $|\alpha|=0$ we have $x_{\alpha}^{j}=x^{j}$. The class $\mathfrak{H}_{k}$ with

$$
(x, y)_{k}=\int_{T} x_{\alpha}^{j}(t) \dot{y}_{\alpha}^{j}(t) d t \quad(|\alpha| \leqq k, j \leqq n)
$$

forms a Hilbert space, as is easily seen with the help of Lemma 5.3. Here and elsewhere a repeated index in a term denotes summation with respect to that index unless otherwise specified or implied. The norm in $\mathfrak{F}_{k}$ will be denoted by $\|x\|_{k}$. When $k$ is held fast as is normally done, we shall use the simpler notations $\mathcal{F},(x, y),\|x\|$ in place of $\mathcal{F}_{k},(x, y)_{k},\|x\|_{k}$. If we wish to denote the dependence of $\mathfrak{H}_{k}$ on $T$ or $n$ we shall use the alternate notations $\mathfrak{H}_{k}(T)$, $\mathcal{K C}_{k}^{n}(T), \mathcal{F}_{k}^{n}$. We are also interested in the case in which the functions $x$ and the scalars in $\mathfrak{H}_{k}$ are restricted to be real. In this event we set $\bar{a}=a$ and replace $-i$ by 1 in the formula (6.1). Although we shall restrict ourselves to the complex case, the proofs are designed so as to be equally valid in the case of reals, whenever it is possible to do so.

Hereafter we shall assume that the set $T$ is the union of a finite number of sets $T$ each of which is the one to one image of an $m$-dimensional interval $S$ under a transformation $t(s)$ which together with its inverse $s(t)$ satisfies a uniform Lipschitz condition. Under this assumption the following theorem can be established.

THEOREM 6.1. The quadratic form

$$
K(x)=\int_{T} x_{\alpha}^{j} b_{\alpha \beta}^{i j} x_{\beta}^{j} d t \quad(|\alpha| \leqq k,|\beta| \leqq k,|\alpha|+|\beta|<2 k, i \leqq n, j \leqq h)
$$


where $b_{\alpha \beta}^{i j}(t)=b_{\beta \alpha}^{H}(t)$ are bounded integrable functions of $t$ on $T$, is w-continuous on $\mathfrak{H C}_{k}$. In particular $\|x\|_{h}^{2}$ with $h<k$ is w-continuous on $\mathfrak{F C}_{k}$ and the quadratic form

$$
H(x)=\int_{T} \int_{T} x_{\alpha}^{i}(s) c_{\alpha \beta}^{i j}(s, t) \tilde{x}_{\beta}^{j}(t) d s d t \quad(|\alpha| \leqq k,|\beta| \leqq k)
$$

is w-continuous on $\mathfrak{F C}_{k}$ if the functions $c_{\alpha \beta}^{i j}(s, t)=\tau_{\beta \alpha}^{i t}(t, s)$ are square integrable on $T \times T$.

The last statement is well known. A simple proof has been given by the author [11, p. 531]. The $w$-continuity of $K$ follows from the $w$-continuity of $\|x\|_{n}^{2}$ for $h<k$. In order to prove that $\|x\|_{h}^{2}(h<k)$ is $w$-continuous, it is sufficient to prove that $\|x\|_{0}^{2}$ is $w$-continuous on $\mathfrak{F}_{1}$. This fact has been established by Morrey in the papers cited above. A simple proof can be made as follows. It is sufficient to treat the case when $n=1$.

Suppose first that $T$ is an interval and let $\left\{x_{q}\right\}$ be a sequence of functions in $\mathfrak{F C}_{1}$ that converges weakly to $x=0$. Then, by Lemma 5.1 , the $h$-averages $x_{q}^{h}(t)$ of $x_{q}(t)$ converge uniformly to $x=0$ for each $h$ on $0<h \leqq 1$. In view of Lemma 5.4 there is a constant $M$ such that

$$
\left\|x_{q}^{h}-x_{q}\right\|_{0} \leqq h M\left\|x_{q}\right\|_{1} .
$$

Hence if we select $N$ so that $\left\|x_{q}\right\|_{1} \leqq N$ we have

$$
\begin{aligned}
\left\|x_{q}\right\|_{0} & \leqq\left\|x_{q}^{h}\right\|_{0}+\left\|x_{q}-x_{q}^{h}\right\|_{0} \leqq\left\|x_{q}^{h}\right\|_{0}+h M N, \\
\limsup _{q=\infty}\left\|x_{q}\right\|_{0} & \leqq h M N
\end{aligned}
$$

for each $h$ on $0<h \leqq 1$. Consequently $\left\|x_{q}\right\|_{0 \rightarrow 0}$, as was to be proved.

Suppose next that $T$ is the one to one image of an interval under a transformation $t(s)$ which together with its inverse $s(t)$ satisfies a uniform Lipschitz condition. Then the Jacobians $j(s), j^{-1}(t)$ of $t(s)$ and $s(t)$-are bounded almost everywhere. There is accordingly a constant $\epsilon>0$ such that $|j(s)| \geqq \epsilon$ almost everywhere on $S$ and $\left|j^{-1}(t)\right| \geqq \epsilon$ almost everywhere on $T$. Under the transformation $t(s)$ a function $x(t)$ in $\mathfrak{H}_{1}(T)$ is transformed into the function $y(s)$ $=x(t(s))$ in $\mathfrak{K}_{1}(S)$ and the chain rule for differentiation holds. Moreover, in view of the uniform Lipschitz condition, there exist positive constants $M$ and $N$ such that for almost all corresponding points $s$ and $t$ we have

$$
M x_{\alpha}(t) \bar{x}_{\alpha}(t) \leqq y_{\alpha}(s) \bar{y}_{\alpha}(s) \leqq N x_{\alpha}(t) \bar{x}_{\alpha}(t), \quad|\alpha|=1 .
$$

It follows that, with $|\alpha|=1$, 


$$
\begin{gathered}
N \int_{T} x_{\alpha} \bar{x}_{\alpha} d t \geqq \int_{S} y_{\alpha} \bar{y}_{\alpha}|j(s)| d s \geqq \epsilon \int_{S} y_{\alpha} \bar{y}_{\alpha} d s \geqq \epsilon^{2} M \int_{T} x_{\alpha} \bar{x}_{\alpha} d t, \\
\int_{T}|x|^{2} d t \geqq \epsilon \int_{S}|y|^{2} d s \geqq \epsilon^{2} \int_{T}|x|^{2} d t .
\end{gathered}
$$

With the help of these inequalities it follows that $x_{q} \rightarrow 0$ in $\mathcal{F}_{1}(T)$ if and only if the corresponding sequence $y_{q} \rightarrow 0$ in $\mathcal{H}_{1}(S)$ and that $\left\|x_{q}\right\|_{0} \rightarrow 0$ if and only if $\left\|y_{q}\right\|_{0} \rightarrow 0$. Thus $\|x\|_{0}^{2}$ is $w$-continuous in $\mathcal{C}_{1}(T)$ as was to be proved.

If $T$ is the union of a finite number of sets $T_{1}, \cdots, T_{p}$ of the type just described, and $x_{q} \rightarrow 0$ in $\mathcal{F}_{1}(T)$, then the section of $x_{q}(t)$ on $T_{h}$ converges weakly to zero on $\mathfrak{F}_{1}\left(T_{h}\right)(h=1, \cdots, p)$. It follows that

$$
\lim _{q=0} \int_{T_{h}}\left|x_{q}(t)\right|^{2} d t=0, \quad h=1, \cdots, p .
$$

Consequently $\left\|x_{q}\right\|_{0}^{2} \rightarrow 0$ as was to be proved.

In the following theorem we shall assume that to each point $t_{0}$ on the boundary of $T$ there is a neighborhood $N$ of $t_{0}$ and an integer $\mu \leqq m$ such that $N \cap \bar{T}$ is the one to one image of the interval

$$
0 \leqq s_{\sigma}<1(\sigma \leqq \mu), \quad-1<s_{r}<1(\mu<\tau)
$$

( $t_{0}$ corresponding to $s=0$ ) under a transformation $t(s)$ which together with inverse $s(t)$ is of class $D^{k}$ and possesses uniformly bounded $k$ th derivatives. The portion of the boundary of $T$ in $N$ corresponds to points $s$ having $s_{\sigma}=0(\sigma \leqq \mu)$. The assumption that the $k$ th derivatives of $t(s)$ and $s(t)$ are uniformly bounded is equivalent to the assumption that they are of class $C^{k-1)}$ and their $(k-1)$ st derivatives are bounded. If $T$ has the properties just described then it will be said to be of class $B^{k}$ and integer $\mu$ will be called the index of $t_{0}$ as a boundary point of $T$.

THEOREM 6.2. Suppose the boundary of $T$ is of class $B^{k}$ and let $B_{k}$ be the class of all $x$ in $\mathfrak{F C}_{k}$ whose kth derivatives are bounded on $T$. The class $B_{k}$ is dense in $\mathfrak{H}_{k}$ and consists of all $x$ in $\mathfrak{H}_{k}$ of class $C^{(k-1)}$ on $T$ whose $(k-1)$ st derivatives are Lipschitzian. Let $\mathcal{H C}_{k 0}$ be the closure in $\mathcal{H}_{k}$ of the class of $x$ in $\mathbb{B}_{k}$ which together with its first $(k-1)$ derivatives vanish on the boundary of $T$. Then $\mathfrak{K C}_{k 0}$ is the closure of the functions of class $C^{(\infty)}$ in $\mathcal{H}_{k}$ whose support set is in $T$.

7. Legendre forms on subspaces of $\mathfrak{H C}=\mathfrak{F}_{k}$. Throughout this section $\mathfrak{A}$ denotes a subspace of $\mathfrak{F}=\mathfrak{H C}_{k}$ and $J$ denotes a quadratic form of the type

$$
J(x)=R(x)+K(x)
$$

where $K(x)$ is $w$-continuous on $\mathfrak{P}$ and 


$$
R(x)=\int_{T} x_{\alpha}^{h}(t) r_{\alpha \beta}^{h j}(t) \dot{x}_{\beta}^{j}(t) d t .
$$

Here $h, j$ are summed from 1 to $n$ and $\alpha=\left(\alpha_{1}, \cdots, \alpha_{m}\right), \beta=\left(\beta_{1}, \cdots, \beta_{m}\right)$ are summed over all indices such that $|\alpha|=|\beta|=k$. The functions $r_{\alpha \beta}^{N}(t)$ are assumed to be continuous on $\bar{T}$ and to satisfy the relation $\bar{r}_{\alpha \beta}^{h j}=r_{\beta \alpha}^{\text {h }}$ on $T$.

THEOREM 7.1. Let $\mathfrak{A}$ be a subspace of $\mathfrak{J C}_{k}$ and let $\mathfrak{A}_{\mathrm{c}}$ be the class of all $x$ in $\mathfrak{A}$ whose support set have diameters at most $\epsilon$. If $J$ is a Legendre form on $\mathfrak{A}$, there exist positive numbers $\epsilon$ and $h$ such that the inequality

$$
J(x) \geqq h\|x\|^{2}
$$

holds for all $x$ in $\mathfrak{A}_{e}$.

Suppose that the conclusion in the theorem is false. Then there exists a sequence $\left\{x_{q}\right\}$ such that $x_{q}$ is in $\mathfrak{A}_{1 / q}$ and

$$
J\left(x_{q}\right)<(1 / q)\left\|x_{q}\right\|^{2} .
$$

We can suppose that the sequence $\left\{x_{q}\right\}$ has been chosen so that $\left\|x_{q}\right\|=1$. Since $\left\{x_{q}\right\}$ then converges weakly in subsequence, we can suppose that it converges weakly to a vector $x_{0}$ in $\mathfrak{A}$. Since the diameter of the support set for $x_{q}$ is at most $1 / q$ it follows that $x_{0}=0$. Since $J$ is $w / s$-continuous we have

$$
0=J\left(x_{0}\right) \leqq \liminf _{q=\infty} J\left(x_{q}\right) \leqq \limsup _{q=\infty} J\left(x_{q}\right) \leqq 0 .
$$

Consequently $J\left(x_{q}\right) \rightarrow 0$ and hence $x_{q} \Rightarrow 0$, since $J$ is a Legendre form. This is impossible, since $\left\|x_{q}\right\|=1$ and the theorem is established.

As a converse to Theorem 7.1 we have the following

THEOREM 7.2. Suppose that the subspace $\mathfrak{A}$ of $\mathfrak{F}$ has the property that if $x$ is in $\mathfrak{A}$ so also is $\rho x$ for every function $\rho$ of class $C^{\infty}$. Suppose further that for each point $t_{0}$ in $\bar{T}$ there is a neighborhood $N$ of $t_{0}$ and a constant $h>0$ such that the inequality (7.3) holds for every $x$ in $\mathfrak{A}$ whose support set is in $N$. Then $J$ is a Legendre form on $\mathfrak{A}$.

For, in view of our hypotheses we may cover $\bar{T}$ by a finite number of spheres $N_{1}, \cdots, N_{p}$ having the property that for each sphere $N_{j}$ there is a constant $h_{j}>0$ such that (7.3) holds with $h=h_{j}$ for every $x$ in $\mathfrak{A}$ whose support set is in $N_{j}$. Let $h$ be the least of the numbers $h_{1}, \cdots, h_{p}$. Select functions $\rho_{1}, \cdots, \rho_{p}$ of class $C^{\infty}$ such that the support set of $\rho_{j}$ is in $N_{j}$ and such that $\rho_{1}^{2}+\cdots+\rho_{p}^{2}=1$ on $T$. Set

$$
Q(x)=J(x)-h\|x\|^{2}, \quad P(x)=Q\left(\rho_{1} x\right)+\cdots+Q\left(\rho_{p} x\right) .
$$

Since the support set of $\rho_{j} x$ is in $N_{j}$ it follows that $Q\left(\rho_{j} x\right) \geqq 0$ on $\mathfrak{A}$ and hence 
that $P(x) \geqq 0$ on $\mathfrak{A}$. Since $\rho_{1}^{2}+\cdots+\rho_{p}^{2}=1$ the terms in the difference

$$
K_{1}(x)=Q(x)-P(x)
$$

arising from $R(x)$ and $\|x\|_{k}^{2}$ involving products of $k$ th derivatives $x_{\alpha}^{i} \bar{x}_{\beta}^{j}$ cancel. It follows from Theorem 6.1 that $K_{1}$ is w-continuous. The quadratic form $Q$ is the sum of a nonnegative form $P$ and a $w$-continuous form $K_{1}$ and is accordingly wls-continuous. The quadratic form $J$ is therefore a Legendre form, by Theorem 3.1, as was to be proved.

For each point $t_{0}$ in $\bar{T}$ let

$$
R\left(x, t_{0}\right)=\int_{T} r_{\alpha \beta}^{i j}\left(t_{0}\right) x_{\alpha}^{i}(t) \bar{x}_{\beta}^{j}(t) d t
$$

where $\alpha, \beta, i, j$ are summed as in the definition of $R(x)$ in $J$. With the help of this quadratic form we can prove the following:

Theorem 7.3. Suppose that $\mathfrak{A}$ has the property described in Theorem 7.2. Then $J$ is a Legendre form on $\mathfrak{A}$ if and only if to each point $t_{0}$ in $\bar{T}$ there is a neighborhood $N$ of $t_{0}$ such that the quadratic form $R\left(x, t_{0}\right)$ defined by (7.5) is positive definite on the class of all $x$ in $\mathfrak{A}$ whose support set is in $N$.

Since $J(x)$ is a Legendre form if and only if $R(x)$ is a Legendre form we can suppose that $J(x)=R(x)$. Suppose therefore that $R(x)$ is a Legendre form. Let $t_{0}$ be a point in $\bar{T}$. In view of Theorem 7.1 there exist positive constants $h$ and $\epsilon$ such that the inequality

$$
R(x) \geqq 2 h\|x\|^{2}
$$

for all $x$ in $\mathfrak{A}$ whose support set is in the $\epsilon$-neighborhood of $t_{0}$. Let $N$ be a smaller neighborhood of $t_{0}$ chosen so that

$$
\left|\int_{N}\left[r_{\alpha \beta}^{i j}(t)-r_{\alpha \beta}^{i j}\left(t_{0}\right)\right] x_{\alpha}^{i}(t) \bar{x}_{\beta}^{j}(t) d t\right|<h\|x\|^{2}
$$

for all $x$ in $\mathfrak{A}$. Combining this result with (7.6) we see that

$$
R\left(x, t_{0}\right) \geqq h\|x\|^{2}
$$

holds for all $x$ in $\mathfrak{A}$ whose support set is in $N$.

Suppose conversely that for each point $t_{0}$ in $\bar{T}$ there is a neighborhood $N$ and a constant $h>0$ such that

$$
R\left(x, t_{0}\right) \geqq 2 h\|x\|^{2}
$$

for all $x$ in $\mathfrak{A}$ whose support set is in $N$. Diminish $N$ if necessary so that (7.7) holds. Then $R(x)$ is positive definite on the class of all $x$ in $\mathfrak{A}$ whose support set is in $N$. It follows from Theorem 7.2 that $R(x)$ is a Legendre form. This proves the theorem. 
8. Conditions of Legendre. Let

$$
r(t, \xi, \zeta)=r_{\alpha \beta}^{h j} \xi^{\alpha} \xi^{\beta} \zeta^{h} \xi^{j}, \quad|\alpha|=|\beta|=k, \quad h \leqq n, \quad j \leqq n,
$$

where $\alpha=\left(\alpha_{1}, \cdots, \alpha_{m}\right), \beta=\left(\beta_{1}, \cdots, \beta_{m}\right)$ and $\xi^{\alpha}$ is the product $\xi_{1}^{\alpha_{1}} \xi_{2}^{\alpha_{2}} \cdots \xi_{m}^{\alpha_{m}}$ of powers of $\xi_{1}, \cdots, \xi_{m}$. The quadratic form $J$ defined by (7.1) will be said to satisfy the condition I of Legendre on $T$ if the inequality

$$
r(t, \xi, \zeta) \geqq 0
$$

holds on $T$ for all sets $(\xi, \zeta)$ such that $\xi=\left(\xi_{1}, \ldots, \xi_{m}\right)$ is real and $\zeta=\left(\zeta^{1}, \cdots, \zeta^{n}\right)$ is complex. The quadratic form $J$ will be said to satisfy the condition $\mathrm{I}^{\prime}$ in $T$ if there is a constant $\epsilon>0$ such that the inequality

$$
r(t, \xi, \zeta) \geqq \epsilon|\xi|^{2 k}|\zeta|^{2}
$$

holds on $T$ if $\xi$ is real and $\zeta$ is complex. Here $|\xi|=\left(\xi_{0} \xi_{\sigma}\right)^{1 / 2},|\zeta|$ $=\left(\zeta^{i} \zeta^{j}\right)^{1 / 2}(\sigma=1, \cdots, m ; j=1, \cdots, n)$. If the coefficients $r_{\alpha \beta}^{k j}$ are real, the numbers $\zeta$ can be restricted to be real. It is clear that the condition (8.3) is the Legendre condition I for

$$
J(x, \epsilon)=J(x)-\epsilon\|x\|^{2} .
$$

Recall that a function $x$ in $3 C$ whose $k$ th derivatives are bounded is of class $C^{(k-1)}$ on $T$ and has Lipschitzian $(k-1)$ st derivatives on $T$. Let $\mathcal{K}$ be the closure in $\mathfrak{H C}$ of all functions of this type which together with their partial derivatives of order $\leqq k-1$ vanish on the boundary of $T$. We shall refer to $\mathcal{K}$ as the class of functions in $\mathcal{K}$ which together with its derivatives of order $<k$ vanish on the boundary of $T$. It can be shown that if a function $x$ in $\mathscr{K}$ is extended to be zero on the complement of $T$, then $x$ is of class $D^{(k)}$ on every interval in $t$-space. In view of our assumption on the boundary of $T$, the class $\varkappa$ can be shown to be the closure of all $x$ in $\mathcal{K}$ of class $C^{\infty}$ whose support set is in $T$.

THEOREM 8.1. Let $\mathfrak{K}$ be the set of all $x$ in $3 C$ which together with its derivatives of order $<k$ vanish on the boundary of $T$. The quadratic form $J$ is a Legendre form on $K$ if and only if it satisfies the Legendre condition $\mathrm{I}^{\prime}$ on $T$. It is wlscontinuous on $\mathcal{K}$ if and only if it satisfies the Legendre condition I on $T$.

Suppose first that $J$ is a Legendre form on $\varkappa$. We shall show that the Legendre condition I holds. To this end let $t_{0}$ be a point in $T$ and suppose there exist real numbers $\xi=\left(\xi_{1}, \cdots, \xi_{m}\right)$ and complex numbers $\zeta=\left(\zeta^{1}, \cdots, \zeta^{m}\right)$ such that

$$
r\left(t_{0}, \xi, \zeta\right)<0 .
$$

Given a neighborhood $N$ of $t_{0}$ in $T$ let $\rho(t)$ be a nonzero function of class $C^{\infty}$ whose support set is in $N$. Let $\lambda$ be a real number and set 


$$
x^{i}(t)=\rho(t) \zeta^{i} e^{i \lambda t \xi}, \quad t \xi=t_{1} \xi_{1}+\cdots+t_{m} \xi_{m} .
$$

For this function the quadratic form $R\left(x, t_{0}\right)$ defined by (7.5) is expressible as a polynomial

$$
R\left(x, t_{0}\right)=\lambda^{2 k} A_{0}+\lambda^{2 k-1} A_{1}+\cdots+A_{2 k}
$$

in $\lambda$, where $A_{0}, A_{1}, \cdots, A_{2 k}$ are constants and

$$
A_{0}=r\left(t_{0}, \xi, \zeta\right) \int_{T} \rho(t)^{2} d t<0 .
$$

Since $A_{0}<0$ the number $\lambda$ can be chosen so large that $R\left(x, t_{0}\right)<0$. Since $N$ is an arbitrary neighborhood of $t_{0}$, this is impossible, by Theorem 7.3. It follows that $J$ satisfies condition I. One can obtain the same conclusion by the use of the function

$$
x^{i}(t)=\rho(t) \zeta^{i} \cos \lambda t \xi
$$

in place of the function (8.5). Our conclusion is therefore valid also in the realvalued case.

If $J$ is a Legendre form on $\nVdash$ so also is the quadratic form $J(x, \epsilon)$ defined by (8.4) for $\epsilon$ sufficiently small and positive. Consequently (8.3) holds with this choice of $\epsilon$, that is, $J(x)$ satisfies the condition $\mathrm{I}^{\prime}$. If $J(x)$ is wls-continuous, then $J(x, \epsilon)$ is a Legendre form for all $\epsilon<0$. Consequently (8.3) holds for all $\epsilon<0$. Hence (8.2) holds. A wls-continuous form therefore satisfies the condition I of Legendre.

Suppose next that $J$ satisfies the condition I of Legendre, and that the coefficients $r_{\alpha \beta}^{h y}$ are constants. We shall show that $R(x) \geqq 0$. To this end let $x$ be a function in $\pi$ and extend $x$ to be identically zero on the complement of $T$. One can restrict $x$ to have bounded $k$ th derivatives, if one wishes to do so. Let $\hat{x}^{i}$ denote the Fourier transform

$$
\hat{x}^{i}=(2 \pi)^{-m / 2} \int_{-\infty}^{\infty} e^{-i t \xi_{x^{i}}(t) d t} \quad\left(t \xi=t_{1} \xi_{1}+\cdots+t_{m} \xi_{m}\right)
$$

of $x^{i}$. By integration by parts it is seen that the Fourier transform of $x_{\alpha}^{j}$ is $\xi^{\alpha} \hat{x}^{i}$. Hence

$$
\left.R(x)=\int_{-\infty}^{\infty} r_{\alpha j}^{h j} x_{\alpha}^{h} \bar{x}_{\beta}^{j} d t=\int_{-\infty}^{\infty} r_{\alpha \beta}^{h j} \xi^{\alpha} \xi^{\beta} \hat{x}^{h-j} \hat{x}^{j}\right) d \xi \geqq 0,
$$

as was to be proved. The result just given was established by van Hove, by the same argument for the case $k=1$, and has been given by the various writers on partial differential equations.

Suppose now that $J$ satisfies the condition I' of Legendre and select $\epsilon>0$ so that (8.3) holds as stated. Then by the result just obtained the inequality 


$$
R\left(x, t_{0}\right)=\int_{T}^{h j} r_{\alpha \beta}^{h j}\left(t_{0}\right) x_{\alpha}^{h} \bar{x}_{\beta}^{j} d t \geqq \epsilon \int_{T} x_{\alpha}^{j} \bar{x}_{\beta}^{j} d t,|\alpha|=|\beta|=k,
$$

holds on $\mathcal{K}$. The integral on the right differs from $\|x\|^{2}$ by a $w$-continuous form and is accordingly a Legendre form. Since it is positive on $\mathfrak{K}$, it is positive definite. Hence $R\left(x, t_{0}\right)$ is positive definite on $\mathcal{K}$. By virtue of Theorem 7.3 , it follows that $J(x)$ is a Legendre form on $\Re$, as was to be proved.

Finally, if $J$ satisfies the condition I of Legendre, then, for $\epsilon<0$, the quadratic form $J(x, \epsilon)$ given by (8.4) satisfies the condition $I^{\prime}$ of Legendre. Hence $J$ is $w l s$-continuous on $\mathcal{K}$, by virtue of Theorem 3.1. This completes the proof of Theorem 8.1.

Theorem 8.2. Suppose that $m=1$. Then $J$ is a Legendre form on $\mathfrak{T C}$ if and only if it satisfies condition $\mathrm{I}^{\prime}$ of Legendre. Moreover, $J$ is wls-continuous on $\mathrm{HC}$ if and only if it satisfies condition I of Legendre.

This result follows because $\mathfrak{H C}$ differs from $\mathcal{K}$ by a subspace of finite dimension.

Following terminology used in the calculus of variations, a function $x$ in $\mathfrak{H C}$ will be called an extremal for $J$ if it is $J$ orthogonal to the subclass $\mathfrak{K}$ of $\mathfrak{H C}$ described above. Combining Theorems 3.6 and 8.1 we obtain the following extension of the problem of Dirichlet:

TheOREM 8.3. Suppose that $J$ satisfies the condition $\mathrm{I}^{\prime}$ of Legendre. Given a function $x$ in 3 there is an extremal $y$ having the same boundary values (in the sense that $x-y$ is in $\mathfrak{K}$ ) if and only if $x$ is J-orthogonal to $\mathfrak{K} \cap \mathcal{K}^{J}$.

The quadratic form $J$ will be said to satisfy the Legendre condition II if it satisfies condition I of Legendre and if at each boundary point $t_{0}$ of $T$ the inequality

$$
r\left(t_{0}, \pi\right)=r_{\alpha \beta}^{h j}\left(t_{0}\right) \pi_{\alpha}^{h} \bar{\pi}_{\beta}^{j} \geqq 0,
$$

$$
|\alpha|=|\beta|=k,
$$

holds for every set $\pi_{\alpha}^{j}$ of complex numbers. It will be said to satisfy the condition $\mathrm{II}^{\prime}$ of Legendre if it satisfies condition $\mathrm{I}^{\prime}$ on $T$ and if there is a constant $\epsilon>0$ such that the inequality

$$
r\left(t_{0}, \pi\right) \geqq \epsilon \pi_{\alpha}^{j} \bar{\pi}_{\alpha}^{j}
$$

holds at each boundary $t_{0}$ of $T$ for every set $\pi_{\alpha}^{\prime}$ of complex numbers. The condition II is satisfied by certain quadratic forms arising in the theory of partial differential equations. The condition $\mathrm{II}^{\prime}$ is satisfied by $J(x)=\|x\|^{2}$.

THEOREM 8.4. If $J$ satisfies the condition $\mathrm{II}^{\prime}$ of Legendre, then $J$ is a Legendre form on $\mathcal{H C}$. If $J$ satisfies the condition II of Legendre, it is wls-continuous on $\mathcal{H C}$. 
This result follows readily from Theorem 7.3. The condition II' of Legendre is not in general a necessary condition for $J$ to be a Legendre form.

We shall give three further conditions of Legendre which are valid if the boundary is of class $B^{k}$ and each boundary point is of index $\mu=1$ in the sense described in the paragraph preceding Theorem 6.2. Consider now a boundary point $t_{0}$ and let the $t_{1}$-axis be the normal at $t=t_{0}$. It will be convenient to use the variables $\left(\xi, \eta_{1}, \cdots, \eta_{m-1}\right)$ in place of $\left(\xi_{1}, \cdots, \xi_{m}\right)$. Then the characteristic form (8.1) can be written as

$$
r\left(t_{0}, \xi, \eta, \zeta\right)=r_{\alpha \gamma, \beta \delta}^{h j} \xi^{\alpha} \bar{\xi}^{\beta} \eta^{\gamma} \bar{\eta}^{\delta} \zeta^{h} \xi^{j}
$$

where $\alpha, \beta$ represent the numbers $\alpha_{1}, \beta_{1}$ in (8.1) and $\gamma=\left(\gamma_{1}, \cdots, \gamma_{m-1}\right)$, $\delta=\left(\delta_{1}, \cdots, \delta_{m-1}\right), \eta^{\gamma}=\eta_{1}^{\gamma_{1}} \eta_{2}^{\gamma_{2}} \cdots \eta_{m-1}^{\gamma_{m-1}}$. We shall also use the abbreviated notation

$$
r\left(t_{0}, \xi, \eta, \zeta\right)=r_{\alpha \beta}^{h j} \xi^{\alpha} \xi^{\beta} \zeta^{i} \xi^{j}
$$

where

$$
r_{\alpha \beta}^{h j}=r_{\alpha \gamma, \beta \delta}^{h j} \eta^{\gamma} \bar{\eta}^{\delta} .
$$

In these formulas the coefficients are to be evaluated at $t=t_{0}$. With these notations in mind we can state the following further modification of the Legendre condition $\mathrm{I}^{\prime}$.

The quadratic form $J(x)$ will be said to satisfy the condition III' $^{\prime}$ of Legendre if

(a) The condition $\mathrm{I}^{\prime}$ of Legendre holds.

(b) At each boundary point $t_{0}$ there is a constant $\epsilon>0$ such that the condition

$$
r\left(t_{0}, \xi, \eta, \zeta\right) \geqq \epsilon\left[|\xi|^{2}+|\eta|^{2}\right]^{k}|\zeta|^{2}
$$

holds for all complex numbers $(\xi, \eta, \zeta)$ with $\eta$ real, where $r\left(t_{0}, \xi, \eta, \zeta\right)$ is given by (8.8).

(c) At each boundary point $t_{0}$ the inequality

$$
r_{\alpha \beta}^{h j} \pi_{\alpha}^{h \bar{\pi}_{\beta}^{j}} \geqq 0 \quad(\alpha, \beta=1, \cdots, m ; i, j=1, \cdots, n)
$$

holds for all complex numbers $\pi_{\alpha}^{n}$ for all $\eta$ with $|\eta|=1$ where $r_{\beta \alpha}^{h y}$ is given by (8.10).

We have the following extension of a result given by Aronszajn [1]:

TheOREM 8.5. Suppose that the boundary of $T$ is of class $B^{k}$ and each boundary point $t_{0}$ if of index $\mu=1$. If the condition III' of Legendre holds then $J(x)$ is a Legendre form on $\mathrm{HC}$. 
This result follows from Theorem 7.3 and Theorem 10.2 to be given in $\$ 10$. The condition III' $^{\prime}$ as stated is not in general a necessary condition for $J(x)$ to be a Legendre form on $3 C$. The condition (8.11) however is a necessary condition, as can be seen by the use of Theorem 9.4 in the next section.

Consider next a boundary point $t_{0}$ at which the Legendre condition $\mathrm{I}^{\prime}$ holds. Then the determinant

$$
\Delta(\xi, \eta)=\operatorname{det}\left(r_{\alpha \beta}^{h j} \xi^{\alpha+\beta}\right),
$$

where $r_{\alpha \beta}^{\text {nj }}$ is given by $(8.10)$, is different from zero for all real numbers $(\xi, \eta)$ with $|\eta|=1$. Let $C^{h i}$ be the cofactor of $r_{\alpha \phi}^{h j} \xi^{\alpha+\beta}$ in this determinant. Let $v_{0}^{n}, \cdots, v_{k-1}^{n}$ be arbitrary constants and set

$$
\begin{aligned}
& V_{\alpha}^{h}=\xi^{\alpha-1} v_{0}^{h}+\xi^{\alpha-2} v_{1}^{h}+\cdots+v_{\alpha-1}^{h}, \\
& V^{h}=\bar{C}^{h \sigma_{0}^{\alpha}} \xi^{\alpha j} \bar{\tau}_{\alpha \beta}^{j} V_{\beta}^{j} \Delta^{-1}, \\
& R_{\alpha}^{h}=V_{\alpha}^{h}-\xi^{\alpha} V^{h} .
\end{aligned}
$$$$
(g=1, \cdots, n)
$$

As will be seen in $\S 10$ below, $R_{\alpha}^{n}$ is a rational function of $\xi$ of degree $\leqq-1$. The quadratic form

$$
N\left(v ; \eta, t_{0}\right)=\int_{-\infty}^{\infty} R_{\alpha}^{h} r_{\alpha \beta}^{h j} \bar{R}_{\beta}^{j} d \xi
$$

in $v_{0}^{5}, \cdots, v_{k-1}^{s}$ is therefore well defined. The quadratic form $J(x)$ will be said to satisfy the condition IV' of Legendre if

(a) The condition I' of Legendre holds.

(b) At a boundary point $t_{0}$ the quadratic form $N\left(v ; \eta, t_{0}\right)$ defined by (8.13) is positive definite in $v_{0}^{s}, \cdots, v_{k-1}^{s}$ for each set of real numbers $\eta=\left(\eta_{1}, \cdots, \eta_{m-1}\right)$ with $|\eta|=1$.

THEOREM 8.6. Suppose that the boundary of $T$ is of class $B^{k}$ and each boundary point is of index $\mu=1$. The quadratic form $J(x)$ is a Legendre form on $3 \mathrm{C}$ if and only if condition $\mathrm{IV}^{\prime}$ of Legendre holds.

This result follows from Theorem 10.1 given in $\$ 10$ below with the help of Theorem 7.3. A necessary condition at boundary points of index $\mu>1$ will be given in Theorem 9.3.

There is an intermediate condition of Legendre which will insure that $J(x)$ is a Legendre form on certain subclasses of $\mathcal{H}$. This condition will be called the condition $\mathrm{V}^{\prime}$ of Legendre and is defined as follows:

(a) The condition I' of Legendre holds.

(b) At each boundary point $t_{0}$ the condition (8.12) holds, as stated.

(c) To each boundary point $t_{0}$ there is an integer $r\left(t_{0}\right) \leqq k$ such that given a set of polynomials $Z^{i}(\xi)$ of $\xi$, not vanishing simultaneously, the polynomials $\bar{r}_{\alpha \beta}^{n} \xi^{\beta} Z^{j}(\xi)$ in $\xi$ have, for each $\eta$ with $|\eta|=1$, at most $r\left(t_{0}\right)$ common zeros 
(counting multiplicities) on each side of the real axis.

Let $B$ be the subclass of all functions in $\mathcal{H C}$ which together with its derivatives of orders $<r\left(t_{0}\right)$ vanish on the part of the boundary lying in some neighborhood of a boundary point $t_{0}$. We have the following

THEOREM 8.7. Suppose that the boundary of $T$ is of class $B^{k}$ and that each boundary point $t_{0}$ is of index $\mu=1$. If the Legendre condition $\mathrm{V}^{\prime}$ holds, then $J(x)$ is a Legendre form on the subspace $B$ of $\pi C$ described above.

This result is an extension of a result given by $M$. Schechter for the case $n=1$. It follows from Theorem 7.3 combined with Theorem 10.3 below.

9. A case with constant coefficients. In order to establish Theorems $\mathbf{8 . 4}$, 8.6 , and 8.7 it is sufficient, by Theorem 7.3 , to study the behavior of $J(x)$ on the class of functions in $\mathcal{H C}$ which vanish exterior to a neighborhood of a boundary point $t_{0}$. By a transformation of variables the portion of $T$ in such a neighborhood can be taken to be an interval. Consequently, we may take $T$ itself to be an interval. Moreover, by Theorem 7.3 we can suppose that $J(x)$ is of the form

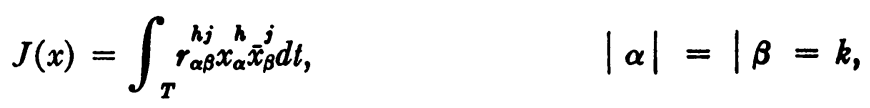

where the coefficients $r_{\alpha \beta}^{h s}$ are constants. This case will be studied in the next three sections. It will be convenient to change our notations somewhat and use $\left(s_{1}, \cdots, s_{\mu}, t_{1}, \cdots, t_{v}\right)$ to denote a point of $T$ in place of $\left(t_{1}, \cdots, t_{m}\right)$. We shall assume that $T$ is the closed interval

$$
0 \leqq s_{\sigma} \leqq b, \quad-b \leqq t_{\tau} \leqq b \quad(\sigma \leqq \mu, \tau \leqq \nu)
$$

and write $x^{i}(s, t)$ in place of $x^{j}(t)$. Let $\alpha=\left(\alpha_{1}, \cdots, \alpha_{\mu}\right), \beta=\left(\beta_{1}, \cdots, \beta_{\mu}\right)$, $\gamma=\left(\gamma_{1}, \cdots, \gamma_{v}\right), \delta=\left(\delta_{1}, \cdots, \delta_{v}\right)$. The partial derivatives of $x^{j}$ will be denoted by

$$
x_{\alpha \gamma}^{j}=(-i)^{|\alpha|+|\gamma|} \frac{\partial^{|\alpha|+|\gamma|} x^{j}}{\partial s_{1}^{\alpha_{1}} \cdots \partial s_{\mu}^{\alpha} \partial t_{1}^{\gamma_{1}} \cdots \partial t_{\nu}^{\gamma}} .
$$

Then $J(x)$ takes the form

$$
J(x)=\int_{0}^{b} \int_{-b}^{b} r_{\alpha \gamma, \beta \delta}^{h j} x_{\alpha \gamma}^{h} \bar{x}_{\beta \delta}^{j} d s d t, \quad|\alpha|+|\gamma|=|\beta|+|\delta|=k .
$$

The characteristic form (8.1) is now

$$
r(\xi, \eta, \zeta)=r_{\alpha \gamma, \beta \delta}^{h j} \xi^{\alpha} \bar{\xi}^{\beta} \eta^{\gamma} \bar{\eta}^{\delta} \zeta^{h} \xi^{j}=r_{\alpha \beta}^{h j}(\eta) \xi^{\alpha} \xi^{\beta} \zeta^{h} \xi^{j}
$$

where 


$$
r_{\alpha \beta}^{h j}(\eta)=r_{\alpha \gamma, \beta \delta \eta^{\prime} \bar{\eta}}^{h j}
$$

We shall assume throughout this section that $J$ satifies the Legendre condition $I^{\prime}$, that is, that there is a constant $\epsilon>0$ such that

$$
r(\xi, \eta, \zeta) \geqq \epsilon\left[|\xi|^{2}+|\eta|^{2}\right]^{k}|\zeta|^{2}
$$

holds for all real numbers $(\xi, \eta)$ and complex numbers $\zeta$. Let $\mathfrak{A}_{b}$ be the class of all $x$ in $\mathfrak{H C}$ such that $x_{\alpha \gamma}^{\sigma}(s, t)=0,|\alpha|+|\gamma|<k$ holds on each face of $T$ defined by one of the relations $t_{\tau}=b, t_{\tau}=-b, s_{0}=b$. Extend the function in $\mathscr{U}_{b}$ so that $x^{j}(s, t)=0$ on the complement of $T$. Then $x^{j}(s, t)$ is of class $D^{k}$ on the infinite interval

$$
0 \leqq s_{\sigma}<\infty, \quad-\infty<t_{r}<+\infty
$$

and $x_{\alpha \gamma}^{J}$ are square integrable on this set. We may accordingly write $J$ in the form

$$
J(x)=\int_{0}^{\infty} \int_{-\infty}^{\infty} r_{\alpha \gamma, \beta \delta}^{h j} x_{\alpha \gamma}^{h} \bar{x}_{\beta \delta}^{j} d s d l
$$

It is easily seen that we can restrict the inner product on $\mathfrak{A}_{b}$ to be of the simpler form

$$
(x, y)=\int_{0}^{\infty} \int_{-\infty}^{\infty} x_{\alpha \gamma}^{j} \bar{y}_{\alpha \gamma}^{j} d s d t, \quad|\alpha|+|\gamma|=k .
$$

Finally, let $\mathfrak{A}_{\infty}$ be the closure of the union of the classes $\mathfrak{A}_{b}(0<b<\infty)$ under the norm just described.

We shall be interested in the behavior of $J$ on certain subclasses of $\mathfrak{A}_{b}$ obtained by imposing restrictions on the faces $s_{\sigma}=0(\sigma=1, \cdots, \mu)$. To this end observe that the boundary values of $x_{\alpha \gamma}^{j},|\alpha| \leqq|\gamma|<k$, are well defined almost everywhere on each of these faces. In the present paper we shall assume that the boundary conditions are such that if $x^{i}(s, t)$ satisfies the boundary conditions so also does $x^{i}(\lambda s, \lambda t)$ for each positive $\lambda$. With this in mind we can prove the following:

THEOREM 9.1. Let $\mathfrak{B}_{\infty}$ be a subclass of $\mathfrak{A}_{\infty}$ with the property that if $x^{i}(s, t)$ is in $B_{\infty}$ so also is $x^{i}(\lambda s, \lambda t)$ for each positive number $\lambda$. Let $B_{b}=\mathfrak{A}_{b} \cap \bigotimes_{\infty}$. Suppose that $0<b<b^{\prime}<\infty$. Then $J(x)$ is nonnegative, positive, or positive definite on $\mathrm{B}_{b}$ if and only if it has the corresponding property on $\mathrm{B}_{b^{\prime}}$. Moreover, $J(x)$ is nonnegative or positive definite on $B_{b}$ if and only if it has the corresponding property on $B_{\infty}$. The quadratic form $J(x)$ is wlsc-continuous on $B_{b}$ if and only if it is nonnegative on $B_{b}$. It is a Legendre form on $B_{b}$ if and only if it is positive definite on $\beta_{b}$.

Let $x^{j}(s, t)$ be a function in $B_{\infty}$ and set $y^{j}(s, t)=\lambda^{m / 2} x^{j}(\lambda s, \lambda t)$, where $\lambda$ is a positive number. Then, as is easily seen, $J(y)=\lambda^{2 k} J(x),\|y\|=\lambda^{k}\|x\|$. More- 
over, if $x$ is in $B_{b}$ then $y$ is in $B_{b_{r}}$. In view of this fact the first two conclusions in the theorem hold. Since $J$ is a Legendre form on $B_{b^{\prime}}$, there is by Theorem 7.1 a number $b$ such that $J$ is positive definite on $B_{b}$. Consequently, $J$ is a Legendre form on $B_{b}$ if and only if it is positive definite on $B_{b}$. The statement on $w / s$-continuity follows from the last statement by replacing $J(x)$ by $J(x)$ $+\epsilon\|x\|^{2}$.

As a next step let $x^{j}(s, t)$ be a function in $B_{b}$ and let

$$
y^{j}(s, \eta)=(2 \pi)^{-\nu / 2} \int_{-\infty}^{\infty} e^{-i \eta_{\eta} x^{j}}(s, t) d t, \quad \quad t \eta=t_{1} \eta_{1}+\cdots+t_{\nu} \eta_{\nu} .
$$

Then, from the theory of Fourier transforms, we have

$$
J(x)=\int_{0}^{\infty} \int_{-\infty}^{\infty} r_{\alpha \beta}^{h j}(\eta) y_{\alpha}^{h}(s, \eta) \bar{y}_{\beta}^{j}(s, \eta) d \eta d s, \quad|\alpha| \leqq k,|\beta| \leqq k,
$$

where the coefficients $r_{\alpha \beta}^{h j}(\eta)$ are given by (9.3). This suggests the study of the integral

$$
P(y ; \eta)=\int_{0}^{\infty} r_{\alpha \beta}^{h j}(\eta) y_{\alpha}^{h}(s) \dot{y}_{\beta}^{j}(s) d s, \quad|\alpha| \leqq k,|\beta| \leqq k .
$$

To this end let $\mathfrak{C}_{\infty}$ be the class of all functions $y^{j}(s)$ of class $\mathfrak{D}^{k}$ on $0 \leqq s<\infty$ which together with their derivatives of orders $\leqq k$ are square integrable on this domain. Let $\mathfrak{C}_{b}$ be the subclass of $\mathfrak{C}_{\infty}$ of all $y$ that vanish on the complement of $0 \leqq s \leqq b$. Let $D_{b}$ be the subclass of all $y$ in $\mathfrak{C}_{b}$ that satisfy the same boundary conditions as those defining $\Theta_{b}$. Finally set

$$
Q(y ; \eta)=\int_{0}^{\infty}|\eta|^{2 k-2|\alpha|} y_{\alpha}^{i}(s) \bar{y}_{\alpha}^{j}(s) d s .
$$

We have the following:

-THEOREM 9.2. The relation $J(x) \geqq 0$ holds on $B_{b}$ if and only if the inequality $P(y ; \eta) \geqq 0$ holds on $D_{b}$ for each set $\eta=\left(\eta_{1}, \cdots, \eta_{v}\right)$ of real numbers. The quadratic form $J(x)$ is a Legendre form on $B_{b}$ if and only if there is a constant $\epsilon>0$ such that

$$
P(y ; \eta) \geqq \epsilon Q(y ; \eta)
$$

holds on $\mathfrak{D}_{p}$ for each real $\eta$.

The last statement follows from the first by replacing $J(x)$ by $J(x)-\epsilon\|x\|^{2}$ and replacing $P(y ; \eta)$ by $P(y ; \eta)-\epsilon Q(y ; \eta)$. If $P(y ; \eta) \geqq 0$ on $D_{b}$ for each $\eta$ then $J(x) \geqq 0$ on $B_{b}$ by virtue of (9.9).

Suppose next that there is a function $y^{j}(s)$ in $D_{b}$ and a set of real numbers $\eta$ such that $P(y ; \eta)<0$. Observe that if $\lambda>1$ then 


$$
z^{i}(s)=\lambda^{\mu / 2} y^{i}(\lambda s)
$$

has the property that

$$
P(z ; \lambda \eta)=\lambda^{2 k} P(y ; \eta)
$$

as can be seen by substitution. Moreover, $z^{j}(s)$ is in $D_{b / \lambda}$. Consider now the function

$$
x^{j}(s, t)=\lambda^{\mu / 2} y^{j}(\lambda s) e^{-i \lambda t \eta p} \rho(t)
$$

where $\rho(t)$ is a nonnull function of class $C^{\infty}$ whose support set is in $-b \leqq t \leqq b$. Then $J(x)$ is expressible in the form

$$
J(x)=\lambda^{2 k} P(y ; \eta) \int_{-b}^{b} \rho^{2}(t) d t+\lambda^{2 k-1} A_{1}+\cdots+A_{2 k}
$$

where $A_{r}$ is of the form

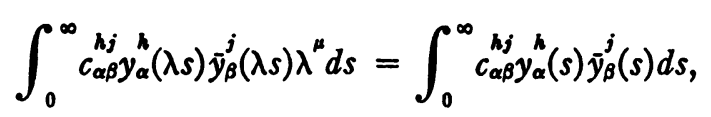

the coefficients $c_{\alpha \beta}^{h y}$ being constants. Since $P(y ; \eta)<0$ we can select $\lambda$ so large that $J(x)<0$. Moreover $x$ is in $B_{b}$. In view of this result it follows that if $J(x) \geqq 0$ on $\mathbb{B}_{b}$, then $P(y ; \eta) \geqq 0$ on $D_{b}$ for each set of real numbers $\eta$.

THEOREM 9.3. Suppose that $0<b \leqq \infty$. Then $P(y ; \eta) \geqq 0$ on $D_{b}$ holds for all real $\eta$ if and only if this inequality holds on $D_{\infty}$ for all real $\eta$ with $|\eta|=1$. There is a constant $\epsilon>0$ such that the inequality (9.12) holds on $D_{b}$ for all real $\eta$ if and only if this inequality holds on $D_{\infty}$ for all real $\eta$ with $|\eta|=1$.

In view of the relations (9.13) and (9.14) it follows that if $0<b<b^{\prime}<\infty$, then $P(y ; \eta) \geqq 0$ on $D_{b}$ for all $\eta$ if and only if $P(y ; \eta) \geqq 0$ on $D_{b}$, for all $\eta$. Hence $P(y ; \eta) \geqq 0$ on $D_{b}$ for all $\eta$ if and only if $P(y ; \eta) \geqq 0$ on $D_{\infty}$ for all $\eta$. Since $P(y ; 0) \geqq 0$ on $D_{\infty}$ if $P(y ; \eta) \geqq 0$ on $D_{\infty}$ for all $\eta \neq 0$, it follows from (9.14) that $P(y ; \eta) \geqq 0$ on $D_{\infty}$ for all $\eta$ if and only if $P(y ; \eta) \geqq 0$ on $D_{\infty}$ for all $\eta$ with $|\eta|=1$. This proves the first statement in the theorem. The second statement is obtained from the first by replacing $P$ by $P-\epsilon Q$.

As a consequence of the last theorem we can prove the following:

THEOREM 9.4. If $J(x)$ is a Legendre form on $\mathfrak{A}_{b}$ then there is an $\epsilon>0$ such that the inequality

$$
r(\xi, \eta, \zeta) \geqq \epsilon\left[|\xi|^{2}+|\eta|^{2}\right]^{k}|\zeta|^{2}
$$

holds for all sets of complex numbers $(\xi, \eta, \zeta)$ such that the $\eta$ 's are real and the imaginary parts of $\xi$ are all of the same sign. Moreover, if $J(x)$ is wls-continuous on $\mathfrak{Q}_{b}$, then the inequality $(9.15)$ with $\epsilon=0$ holds for all sets $(\xi, \eta, \zeta)$ of this type. 
By the arguments used previously the first statement follows from the last statement with $J-\epsilon\|x\|^{2}$ playing the role of $J(x)$. In order to prove the last statement choose a set $(\xi, \eta, \zeta)$ with $\eta$ real and the imaginary parts $\xi^{\prime \prime}$ of $\xi$ being negative. Then $y^{j}=e^{-i \varepsilon \xi \xi^{j}}$ is in $e_{\infty}$ and

$$
P(y ; \eta)=r(\xi, \eta, \zeta) \int_{0}^{\infty} e^{2 \varepsilon \xi \prime \prime} d s
$$

Since $P(y ; \eta) \geqq 0$, by Theorems 9.2 and 9.3 , it follows that $r(\xi, \eta, \zeta) \geqq 0$. Since

$$
r(\xi, \eta, \zeta)=r(-\xi,-\eta, \zeta)
$$

it follows that $r(\xi, \eta, \zeta) \geqq 0$ when the imaginary parts of $\xi$ are positive. By continuity considerations it follows that this inequality holds when the imaginary parts of $\xi$ are nonnegative and nonpositive. This proves the theorem.

10. The case $\mu=1$. In the present section we shall assume that $\mu=1$ and that the class $\mathbb{B}_{b}^{r}$ is the class of all $x$ in $\mathfrak{A}_{b}$ which together with its derivatives of orders $<r$ vanish on face $s=0$ where $r$ is an integer on the range $0 \leqq r \leqq k$. The corresponding subclass $\mathfrak{D}_{b}^{r}$ of $\mathfrak{C}_{b}$ is the class of all $y$ in $\mathfrak{C}_{b}$ such that

$$
y_{0}^{j}(0)=0 \quad(\sigma=0,1, \cdots, r-1) .
$$

It is clear that $\mathbb{B}_{b}^{0}$ coincides with $\mathfrak{A}_{b}$ and $D_{b}^{0}$ coincides with $\mathfrak{C}_{b}$.

Throughout this section it will be convenient to extend the functions $y$ in $\mathfrak{C}_{b}$ (and hence also in $\mathfrak{D}_{b}^{r}$ ) so as to be identically zero on $-\infty<s<0$. The integral $P(y ; \eta)$, defined by $(9.10)$, can be written in the form

$$
P(y ; \eta)=\int_{-\infty}^{\infty} r_{\alpha \beta}^{h j} y_{\alpha}^{h} \bar{y}_{\beta}^{j} d s .
$$

Let $D_{b}^{r-}$ be the class of all functions of the form $y^{j}(-s)$, where $y^{j}(s)$ is in $D_{b}^{r}$. Let $\mathscr{D}_{b}^{r *}$ be the direct sum of the classes $\mathscr{D}_{b}^{r}$ and $\mathfrak{D}_{b}^{r-}$. Similarly set $\mathfrak{C}_{b}^{*}=\mathfrak{C}_{b}+\mathfrak{C}_{b}^{-}$. The class $\mathfrak{D}_{b}^{r^{*}}$ is the class of all $y$ in $\mathfrak{C}_{b}^{*}$ such that

$$
y_{\sigma}^{j}(0+)=y_{0}^{j}(0-)=0 \quad(\sigma=0,1, \cdots, r-1) .
$$

We have the following

LemMa 10.1. The quadratic form $P(y ; \eta)$ is nonnegative, positive, positive definite on $\mathfrak{D}_{b}^{r}$ (on $\mathfrak{C}_{b}$ ) for all real unit vectors $\eta$ if and only if it has the same property on $\mathrm{D}_{b}^{r^{*}}$ (on $\mathfrak{C}_{b}^{*}$ ). The quadratic form $J(x)$ is positive definite on $\mathfrak{A}_{b}$ if and only if $P(y ; \eta)$ is positive definite on $\mathrm{e}_{b}^{*}$ for real unit vectors $\eta$. The quadratic form $J(x)$ is positive definite on $B_{b}^{r}$ if and only if $P(y ; \eta)$ is positive definite on $\mathcal{D}_{b}^{r^{*}}$ for all real unit vectors $\eta$. 
A function $x$ in $D_{b}^{r^{*}}$ is of the form $x^{i}(s)=y^{i}(s)+z^{i}(-s)$, where $y^{i}(s)$ and $z^{j}(s)$ are in $D_{b}^{r}$. Moreover

$$
P(x ; \eta)=P(y ; \eta)+P(z ;-\eta), \quad \quad\|x\|^{2}=\|y\|^{2}+\|z\|^{2} .
$$

It follows that the first statement in the lemma holds. The remaining two statements follow from Theorems 9.2 and 9.3 .

Let $y$ be an arc in $\mathfrak{C}_{b}^{*}$. The quantities

$$
v_{\alpha}^{j}=i(2 \pi)^{-1 / 2}\left[y_{\alpha}^{j}(0+)-y_{\alpha}^{j}(0-)\right] \quad(0 \leqq \alpha<k)
$$

will be taken as a measure of the discontinuity of $y_{\alpha}^{J}(s)$ at $s=0$. As before let $y^{j}$ denote the Fourier transform

$$
\hat{y}^{j}=(2 \pi)^{-1 / 2} \int_{-\infty}^{\infty} e^{-i s \xi y^{j}(s) d s}
$$

of $y^{i}$. The Fourier transforms $\hat{y}_{\alpha}^{j}$ of $y_{\alpha}^{j}$ can be written in the form

$$
y_{\alpha}^{j}=\xi^{\alpha} y^{j}+V_{\alpha}^{j}
$$

where

$$
V_{\alpha}^{h}=V_{\alpha}^{h}(\xi, v)=\xi_{v_{0}^{\alpha-1}}^{h}+\xi_{v_{1}{ }^{\alpha-2}}^{h}+\cdots+y_{\alpha-1}^{h} .
$$

It will be convenient to write the Fourier transform $\hat{y}_{\alpha}^{y}$ in a somewhat different form. To this end recall that, by virtue of our assumption that the condition $I^{\prime}$ of Legendre holds, the determinant

$$
\Delta(\xi, \eta)=\operatorname{det}\left(\boldsymbol{r}_{\alpha \beta}^{h j} \xi^{\alpha+\beta}\right)
$$

is different from zero for all real numbers $(\xi, \eta)$ with $|\eta|=1$. This determinant is a polynomial of degree $2 n k$ in $\xi$. Let $C^{h j}$ be the cofactor of $r_{\alpha \beta}^{\alpha} \xi^{\alpha+\beta}$ in $\Delta(\xi, \eta)$ and set

$$
\begin{aligned}
W^{h} & =\bar{C}^{h o} \xi_{\bar{\eta}}^{\alpha} \tilde{\tau}_{\alpha \beta}^{o j} V_{\beta}^{j}, \quad V^{h}=W^{h} \Delta^{-1}, \\
R_{\alpha}^{h} & =R_{\alpha}^{h}(\xi, v)=V_{\alpha}^{h}-\xi^{\alpha} V^{h} .
\end{aligned}
$$

Observe that $V^{h}$ has been chosen so that the relation

$$
\xi^{\alpha} r_{\alpha \beta}^{h j} \xi \bar{R}_{\beta}^{j} \equiv 0
$$

is an identity in $\xi, v_{0}^{3}, v_{1}^{s}, \cdots, v_{k-1}^{s}$. Setting

$$
\hat{z}^{h}=\hat{y}^{h}+V^{h}
$$

we obtain formulas 


$$
\begin{gathered}
y_{\alpha}^{h}=\xi^{\alpha} \AA^{h}+R_{\alpha}^{h}, \\
r_{\alpha \beta}^{h j} y_{\alpha}^{h} y_{\beta}^{j}=r_{\alpha \beta}^{h j} \xi^{\alpha+\beta} z^{h-j}+R_{\alpha}^{h} \gamma_{\alpha \beta}^{h j} \bar{R}_{\beta}^{j} .
\end{gathered}
$$

We have the following

Lemma 10.2. Suppose that the Legendre condition I' holds. The functions $R_{\alpha}^{h}$ are rational functions in $\xi$ of degree $\leqq-1$ unless they vanish identically in $\xi$. The arc $z$ defined by

$$
z^{i}(s)=(2 \pi)^{-1 / 2} \int_{-\infty}^{\infty} e^{i \xi \varepsilon \tilde{z}^{j}(\xi) d \xi}
$$

is an arc in $\mathfrak{e}_{\infty}^{*}$ having no discontinuities at $s=0$. Moreover

$$
\begin{aligned}
P(y ; \eta) & =P(z ; \eta)+N(v ; \eta), \\
P(y-z ; \eta) & =N(v ; \eta)
\end{aligned}
$$

where

$$
N(v ; \eta)=\int_{-\infty}^{\infty} R_{\alpha}^{h} T_{\alpha \beta}^{h j} \bar{R}_{\beta}^{j} d \xi .
$$

In order to prove the first statement in the lemma the coefficient $p_{a}^{N}$ of $v_{\sigma}^{n}$ in

$$
W^{h}=V^{h} \Delta=p_{\sigma}^{h j j} v_{\sigma}^{j} \quad(\sigma=0,1, \cdots, k-1)
$$

is the polynomial

$$
p_{\sigma}^{h j}=\bar{C}^{h o} \xi_{\xi}^{\alpha} \bar{r}_{\alpha \gamma}^{h j} \xi^{\gamma-\sigma-1} \quad(\gamma=\sigma+1, \cdots, k)
$$

and is of degree $\leqq 2 n k-\sigma-1$. For each $\sigma$ we have

$$
\Delta \delta^{h j}=p_{\sigma}^{h j} \xi^{\sigma+1}+q_{\sigma}^{h j}
$$

where $\delta^{k i}$ is the Kronecker delta and $q_{\sigma}^{n}$ is of degree at most $2 n k-k+\sigma$. Combining (10.14), (10.16), (10.6) and (10.7) it is found that

$$
\begin{aligned}
W^{k} & =v_{\sigma}^{h} \xi^{-\sigma-1} \Delta-q_{\sigma}^{h j} v_{\sigma}^{j} \xi^{-\sigma-1}, \\
R_{\alpha}^{k} & =-v_{\tau}^{h} \xi^{\alpha-\tau-1}+\Delta^{-1} q_{\sigma}^{h j} v_{\sigma}^{j} \xi^{\alpha-\sigma-1} \quad(\alpha<k, \tau=\alpha, \cdots, k-1), \\
R_{k}^{h} & =\Delta^{-1} q_{\sigma}^{h j} v_{\sigma}^{j} k^{-\sigma-1} .
\end{aligned}
$$

From these formulas it is seen that the functions $R_{\alpha}^{h}$ are rational functions of $\xi$ of degree $\leqq-1$ unless $R_{\alpha}^{h} \equiv 0$. This proves the first statement in the lemma. 
Since $R_{\alpha}^{h}$ and $\hat{y}_{\alpha}^{h}$ are square integrable it follows that $\xi^{\alpha} \xi^{h}$ is square integrable. It follows that the function $z^{j}(s)$ defined by $(10.11)$ is in $\mathcal{C}_{\infty}^{*}$ and ${\varkappa_{\alpha}^{h}}^{h} \xi^{\alpha} \varepsilon^{h}$ is the Fourier transform of $z_{\alpha}$. Consequently $z$ has no discontinuities at $s=0$. The first of the formulas (10.12) follows from (10.10). Using (10.8) and (10.10) we see that

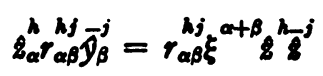

and hence that

$$
P(y-z ; \eta)=P(y ; \eta)-P(z ; \eta) .
$$

It follows that the second formula in (10.12) holds. This proves the lemma.

LEMma 10.3. Suppose that the Legendre condition I' holds. The quadratic form $P(y ; \eta)$ is positive definite on $\mathrm{e}_{\infty}^{*}$ if and only if $N(v ; \eta)$ is positive definite in the variables $v_{0}^{J}, \cdots, v_{k-1}^{u}$ for all real unit vectors $\eta$, where $N(v ; \eta)$ is given by (10.13).

In view of the formulas $(10.12)$ it follows that $P(y ; \eta)$ is positive on $\mathcal{C}_{0}^{*}$ for all real unit vectors $\eta$ if and only if $N(v ; \eta)$ is positive (and hence positive definite) in $v$ for all unit vectors $\eta$. Suppose therefore that $N(v ; \eta)$ is positive definite as stated. Let

$$
r_{\alpha \beta}^{h j}(\eta, \epsilon)=r_{\alpha \beta}^{h j}(\eta)-\epsilon|\eta|^{2 k-2 \alpha} \delta_{\alpha \beta} \delta^{h j}
$$

Then

$$
P(y ; \eta, \epsilon)=\int_{-\infty}^{\infty} r_{\alpha \beta}^{h j}(\eta, \epsilon) y_{\alpha}^{h} \bar{y}_{\beta}^{j}=P(y ; \eta)-\epsilon Q(y ; \eta)
$$

where $Q(y ; \eta)$ is given by (9.11). Observe that $Q(y ; \eta)=\|y\|^{2}$ when $|\eta|=1$. By virtue of the Legendre condition $I^{\prime}$ the inequality

$$
r_{\alpha \beta}^{h j}(\eta, \epsilon) \xi^{\alpha+\beta} \zeta^{h} \xi^{j}>0
$$

holds on interval $0 \leqq \epsilon \leqq \epsilon_{0}$ for all real unit vectors $\eta$, for all real numbers $\xi$ and all complex vectors $\zeta \neq 0$. Let $N(v ; \eta, \epsilon)$ be the quadratic form $(10.13)$ computed with $r_{\alpha \beta}^{h j}(\eta)$ replaced by $r_{\alpha \beta}^{h j}(\eta, \epsilon)$. Then $N(v ; \eta, 0)=N(v ; \eta)$. It follows that if $\epsilon_{0}$ can be chosen sufficiently small then $N(v ; \eta, \epsilon)$ is positive in $v$ for all unit vectors $\eta$ and all $\epsilon$ on $0 \leqq \epsilon \leqq \epsilon_{0}$. Hence $P(y ; \eta, \epsilon)$ is positive on $\mathfrak{C}_{\infty}^{*}$ for these choices $\eta$ and $\epsilon$, that is, $P(y ; \eta)$ is positive definite on $\mathfrak{C}_{\infty}^{*}$ for all unit vectors $\eta$, as was to be proved.

Corollary. Suppose that the Legendre condition I' holds. Then $P(y ; \eta)$ is positive definite on $\mathfrak{C}_{\infty}$ for all unit vectors $\eta$ if and only if it is positive on $\mathfrak{C}_{\infty}$ for all unit vectors $\eta$. 
Combining Lemmas 10.1 and 10.3 we obtain

THEOREM 10.1. Suppose that $\mu=1$ and that the Legendre condition $I^{\prime}$ holds for the quadratic form $J(x)$ defined in \$9. Then $J(x)$ is a Legendre form on the class $\mathfrak{A}_{b}(0<b \leqq \infty)$ if and only if the quadratic form $N(v ; \eta)$ defined by (10.13) is positive definite in the variables $v_{0}^{3}, \cdots, v_{k-1}^{s}$ for all real unit vectors $\eta$.

THEOREM 10.2. Suppose that the inequality

$$
r_{\alpha \beta}^{h j} \pi_{\alpha}^{h} \tilde{\pi}_{\beta}^{j} \geqq 0
$$

holds for all $\pi$ and all real unit vectors $\eta$. Suppose further that there is a constant $\epsilon>0$ such that

$$
r_{\alpha \beta}^{h j} \xi^{\alpha} \xi^{\beta} \zeta^{h} \bar{\zeta}^{j} \geqq \epsilon\left[|\xi|^{2}+|\eta|^{2}\right]^{k}|\zeta|^{2}
$$

holds for all $(\xi, \eta, \zeta)$ with $\eta$ real. Then $J(x)$ is positive definite on the class $\mathfrak{A}_{b}$.

By virtue of Theorem 10.1 it is sufficient to show that the quadratic form $N(v ; \eta)$ is positive definite in $v$ for all $\eta$ with $|\eta|=1$. Suppose that this is not the case and select a $v \neq 0$ and an $\eta$ with $|\eta|=1$ so that $N(v ; \eta)=0$. Then, by (10.18), we have

$$
R_{\alpha}^{h} r_{\alpha \beta}^{h j} \bar{R}_{\beta}^{j}=0
$$

hence

$$
0=r_{\alpha \beta}^{h j} \bar{R}_{\beta}^{j}=r_{\alpha \beta}^{h j} \bar{V}_{\beta}^{j}-r_{\alpha \beta}^{h j} \xi \bar{V}^{j}
$$

holds for all real numbers $\xi$ and hence also for all complex numbers $\xi$. We have accordingly, with $W^{h}=V^{h} \Delta$,

$$
\bar{\xi}^{\alpha} r_{\alpha \beta}^{h j} \bar{V}_{\beta}^{j} \Delta \equiv \xi^{\alpha} r_{\alpha \beta}^{h j} \xi^{\beta} \bar{W}^{j} .
$$

In view of the relation (10.19) the determinant of the coefficients of $\bar{W}^{h}$ is different from zero. Consequently every root of $\Delta(\xi)=0$ is also a root of $\bar{W}^{h}(\xi)=0$ of the same order. This is impossible since the degree of $\Delta$ exceeds that of $\bar{W}^{h}$. This proves the theorem.

The following further result will be useful.

Lemma 10.4. Suppose the condition $I^{\prime}$ of Legendre holds and let $W^{h}$ and $V^{h}$ be defined by (10.7). The relation $W^{h} \equiv 0(h=1, \cdots, n)$ or equivalently the relation $V^{h} \equiv 0(h=1, \cdots, n)$ holds for all real numbers $\xi$ and $a$ given set $\eta$ with $|\eta|=1$ if and only if $v_{\sigma}^{h}=0(\sigma=0,1, \cdots, k-1)$.

This result is an immediate consequence of the first equation in (10.17).

Consider next the subspace $\mathfrak{D}_{\infty}^{r^{*}}$ of $\mathfrak{C}_{\infty}^{*}$ determined by the conditions (10.2). On this class the relations 


$$
v_{\sigma}^{j}=0 \quad(\sigma=0,1, \cdots, r-1)
$$

hold, where $v_{\alpha}^{j}$ is defined by (10.3). It follows that the functions $W^{h}$ defined by (10.7) depend only on $v_{r}^{n}, \cdots, v_{k-1}^{n}$ and are polynomials in $\xi$ of degree at most $2 n k-r-1$, as can be seen by (10.14) and (10.15). For each set $v_{r}^{s}, \cdots, v_{k-1}^{s}$, not all zero, and for each $\eta$ with $|\eta|=1$ we shall define an index $q(v, \eta)$ as follows. Let $F(\xi)$ be the polynomial in $\xi$ whose zeros are the common zeros, counting multiplicities, of the polynomials $W^{1}, \cdots, W^{n}$ in $\xi$ for the given set $(v, \eta)$. We may write $W^{h}$ in the form $W^{h}=Z^{h} F$, where $Z^{h}$ are polynomials that do not vanish simultaneously in $\xi$. The equations

$$
\overline{\boldsymbol{r}}_{\alpha \beta}^{h j} \xi^{\beta} Z^{j}(\xi)=0
$$

have no common real zeros, by virtue of the condition $\mathrm{I}^{\prime}$ of Legendre. Let $q^{+}(v, \eta)$ be the number of common zeros, counting multiplicities, having positive imaginary parts and let $q^{-}(\nu, \eta)$ be the number with negative real parts. Let $q(v, \eta)$ be the larger of $q^{+}(v, \eta)$ and $q^{-}(v, \eta)$. Finally let $q$ be the maximum of the integers $q(v, \eta)$ for all $\eta$ with $|\eta|=1$ and all $v_{r}^{s}, \cdots, v_{k-1}^{s}$, not all zero. We can now establish the following

THEOREM 10.3. Let $r$ be an integer on the range $0 \leqq r<k$. Suppose that the Legendre condition $I^{\prime}$ holds and the inequality (10.18) holds for all $\pi$ and all real unit vectors $\eta$. If the index $q$ defined above does not exceed $r$, then $J(x)$ is positive definite on the class $B_{\infty}^{r}$ of all arcs in $\mathfrak{A}_{\infty}$ which vanish, together with its normal derivatives of orders $<r$, on the face $s=0$.

The proof of this result will be given in the next section. The theorem has been established, with minor modifications, by $M$. Schechter for the case $n=1$. The proof here given is modeled after that given by Schechter [20].

COROLLARY. Let $q^{\prime}$ be the least integer such that for real unit vector $\eta$ and for polynomials $Z^{j}(\xi)$, not vanishing simultaneously, the equations (10.20) have at most $q^{\prime}$ roots with positive imaginary parts and at most $q^{\prime}$ roots with negative imaginary parts. The conclusion of Theorem 10.3 remains valid if the index $q^{\prime}$ is used in place of $q$ in the statement of the theorem.

11. Proof of Theorem 10.3. The proof of Theorem 10.3 is based on a sequence of lemmas, the first of which is the following

LEMMA 11.1. Let $g$ and $r$ be integers such that $g>r>0$. Let $G(\xi)$ be a polynomial of degree $g$ having no real roots and having at most $r$ roots (counting multiplicities) on either side of the real axis. Let $H(\xi)$ be a nonnull polynomial of degree at most $g-r-1$. There is an integer $\sigma(0 \leqq \sigma<r)$ such that

$$
\int_{-\infty}^{\infty} \frac{\xi^{\sigma} H(\xi)}{G(\xi)} d \xi \neq 0 .
$$


This lemma has been established by M. Schechter [20, p. 167].

Lemma 11.2. Suppose that the I egendre condition $\mathrm{I}^{\prime}$ holds and let $V_{\beta_{2}}^{f} W^{i}, \Delta$, $V^{i}=W^{i} \Delta$ be the functions appearing in (10.7). Let $r$ be an integer on the range $0<r<k$. Suppose that for a given set $(v, \eta)$ with

$$
v_{\sigma}^{j}=0(\sigma<r), \quad v_{j}^{k}(r \leqq \rho<k) \text { not all zero, }|\eta|=1
$$

the relations

$$
\bar{r}_{\alpha \beta}^{h j} V_{\beta}^{j}=\bar{i}_{\alpha \beta}^{h j} \xi^{\beta} V^{j}
$$

hold identically in $\xi$ on $-\infty<\xi<\infty$. Let $F(\xi)$ be the greatest common divisor of the polynomials $W^{1}, \cdots, W^{n}$ in $\xi$ and set $Z^{i}=W^{i} / F$. If the polynomials $\tilde{r}_{\alpha \beta}^{n \alpha} \xi^{\beta} Z^{i}$ have at most $r$ common zeros on each side of the real axis, counting multiplicities, then the quantities

$$
u_{o}^{h}=\int_{-\infty}^{\infty} \xi^{\sigma} V^{h} d \xi \quad(\sigma=0,2, \cdots, r-1 ; h=1, \cdots, n)
$$

do not vanish simultaneously for the given set $(v, \eta)$.

Let $K$ be the greatest common divisor of $F$ and $\Delta$ and set $G=\Delta / K$, $L=F / K$. Writing (11.2) in the form

$$
\bar{r}_{\alpha \beta}^{h j} V_{\beta}^{j} G=\bar{r}_{\alpha \beta}^{h j} \xi^{\beta} Z^{j} L,
$$

then $G$ must be a divisor of $\bar{r}_{\alpha \beta}^{n j} \xi^{\beta} Z^{j}$ and hence can have at most $r$ zeros on either side of the axis. By virtue of the Legendre condition $\mathrm{I}^{\prime}$, the polynomial $\Delta$ and hence $G$ has no real zeros. Moreover, since $\Delta$ is of degree $2 n k$ and $W^{i}$ is of degree at most $2 n k-r-1$, it follows that $Z^{i} L$ is of degree at most $g-r-1$, where $g$ is the degree of $G$. Consequently, by Lemmas 11.1 and 10.4, the integrals

$$
\int_{-\infty}^{\infty} \xi^{\sigma} V^{i} d \xi=\int_{-\infty}^{\infty} \xi^{\sigma} \frac{Z^{i} L}{G} d \xi \quad(\sigma=0,1, \cdots, r-1)
$$

do not vanish simultaneously, as was to be proved.

Lemma 11.3. Given an integer $r$ on $0<r<k$ let $D_{\infty}^{* *}$ be the class of all arcs in $\mathfrak{e}_{\infty}^{*}$ such that

$$
y_{\sigma}^{j}(0-)=y_{\sigma}^{j}(0+)=0 \quad(\sigma=0,1, \cdots, r-1) .
$$

The Fourier transform $\hat{y}$ of arc $y$ in $\mathfrak{D}_{\infty}^{r^{*}}$ satisfies the relations

$$
\int_{-\infty}^{\infty} \xi^{\sigma} \hat{y}^{j}(\xi) d \xi=0 \quad(\sigma=0,1, \cdots, r-1) .
$$


If $u_{\sigma}^{k}$ is defined by (11.3), with

$$
v_{\sigma}^{j}=0(\sigma<r), v_{p}^{j}=i(2 \pi)^{-1 / 2}\left[y_{p}^{j}(0+)-y_{p}^{j}(0-)\right] \quad(\rho \leqq r<k),
$$

the integral

$$
I(y ; \eta)=\int_{-\infty}^{\infty}\left\{\tilde{u}_{\sigma}^{h} \xi^{\sigma} y^{n}+u_{\sigma}^{h} \xi^{\sigma} j^{h}\right\} d \xi
$$

is identically zero on $D_{\infty}^{* *}$ for all unit vectors $\eta$.

On the class $D_{\infty}^{r^{*}}$ the Fourier transform $\hat{y}_{\sigma}^{h}$ of $y_{\sigma}^{h}$ is given by $\hat{y}_{\sigma}^{h}=\xi^{\circ} \hat{y}^{h}$, by virtue of (11.4). Using (11.4) and the formula

$$
y_{0}^{h}(s)=(2 \pi)^{-1 / 2} \int_{-\infty}^{\infty} e^{i s \xi} \xi^{\sigma} y^{j}(\xi) d \xi
$$

it is seen that (11.5) holds. Using (11.5) it follows that $I(y ; \eta)=0$ on $D_{\infty}^{r^{*}}$. This proves the lemma.

We are now in a position to prove Theorem 10.3. To this end let $u_{\sigma}^{n}$ be defined by (11.3). The function $u_{\sigma}^{n}$ depends on $v_{r}^{n}, \cdots, v_{k-1}^{n}$ and on $\eta$. Suppose that we have given a particular arc $y$ in $D_{\infty}^{r_{*}^{*}}$ and let $v_{\alpha}^{k} \eta \eta$ satisfy (11.6). Let $S^{h}, z^{h}, \omega^{h}$ be given by the formulas

$$
S^{h}=\bar{C}^{h j} \bar{u}_{\sigma}^{j} \xi^{\sigma}, \quad z^{h}=y^{h}+V^{h}, \quad w^{h}=z-\lambda S^{h}
$$

where $\hat{y}^{h}, V^{h}$ are given by (10.4) and (10.7), $\bar{C}^{h j}$ are the cofactors appearing in (10.7) and $\lambda$ is a real number to be chosen presently. Observe that $S^{h}$ has been chosen so that

$$
\dot{\xi} \bar{u}_{0}^{h}=\bar{r}_{\alpha \beta}^{h j} \xi^{\alpha+\beta} S^{j}
$$

and hence the integral (11.7) takes the form

$$
I(y ; \eta)=2 \int_{-\infty}^{\infty} \operatorname{Re}[r(\xi, \eta, \hat{y}, S)] d \xi
$$

where $\operatorname{Re}(V)$ denotes the real part of $V$ and

$$
r\left(\xi, \eta, \zeta, \zeta_{1}\right)=r_{\alpha \beta}^{h j} \xi^{\alpha+\beta} \zeta^{h} \xi_{1}^{j}
$$

Setting $r(\xi, \eta, \zeta)=r(\xi, \eta, \zeta, \zeta)$, we have the identity

$$
\begin{aligned}
r(\xi, \eta, \hat{w})+2 \lambda \operatorname{Re}[r(\xi, \eta, V, S)]-\lambda^{2} r(\xi, \eta, S) & \\
& =r(\xi, \eta, \hat{z})-2 \lambda \operatorname{Re}[r(\xi, \eta, \hat{y}, S)] .
\end{aligned}
$$

Using (10.10) we obtain the formula 


$$
P(y ; \eta)-2 \lambda I(y ; \eta)=\int_{-\infty}^{\infty} r(\xi, \eta, \hat{z}) d \xi+M(\nu ; \eta)
$$

where

$$
M(v ; \eta)=N(v ; \eta)+\int_{-\infty}^{\infty}\left\{2 \lambda \operatorname{Re}[r(\xi, \eta, V, S)]-\lambda^{2} r(\xi, \eta, S)\right\} d \xi
$$

Using the relation (11.8) and the formula for $S$ it is seen that

$$
M(v ; \eta)=N(v ; \eta)+2 \lambda u_{\sigma}^{h} \bar{u}_{\sigma}^{h}-\lambda^{2} u_{\sigma}^{h} u_{\tau}^{\cdot} \int_{-\infty}^{\infty} \frac{\bar{C}^{h i} \xi^{\sigma+\tau}}{\Delta} d \xi .
$$

Select $\lambda$ so that the quadratic form in $u_{\sigma}^{n}$ defined by the last two terms in this expression is positive definite. We shall show that $M(v ; \eta)$ is a positive definite quadratic form in $v_{r}^{k}, \cdots, v_{k-1}^{h}$ for all $\eta$ with $|\eta|=1$. To this end observe that if $(v, \eta)$ are such that $N(v ; \eta)=0$, then, as in the proof of Theorem 10.2, the relations (11.2) hold. By Lemma 11.2 the quantities $u_{\sigma}^{n}$ are not all zero unless the quantities (11.6) all vanish. Consequently, $M(v ; \eta)$ is positive definite. Since $I(y ; \eta)=0$ on $D_{\infty}^{r^{*}}$, it follows from $(11.6)$ that $P(y ; \eta)$ $>0$ for all $y \neq 0$ in $D_{\infty}^{r^{*}}$ and all $\eta$ with $|\eta|=1$.

It remains to show that $P(y ; \eta)$ with $|\eta|=1$ is positive definite on $D_{\infty}^{r^{*}}$. To this end we compute the formula $(11.9)$ for $P(y ; \eta)-\epsilon Q(y ; \eta)$ in place of $P(y ; \eta)$. This can be carried out by using $r_{\alpha \beta}^{h j}-\epsilon \delta_{\alpha \beta} \delta^{h i}$ in place of $r_{\alpha \beta}^{h j}$. One obtains, on $D_{\infty}^{r^{*}}$, a formula of the form

$$
P(y ; \eta)-\epsilon Q(y ; \eta)=\int_{-\infty}^{\infty} r(\xi, \eta, z, \epsilon) d \xi+M(\nu ; \eta, \epsilon)
$$

where the right-hand members reduce to the corresponding right-hand members of (11.9) when $\epsilon=0$. It follows from continuity relations that there is a constant $\epsilon>0$ such that $M(v ; \eta, \epsilon)$ is a positive definite quadratic form in $v_{r}^{n}, \cdots, v_{k-1}^{n}$ for all $\eta$ with $|\eta|=1$. Moreover by virtue of the Legendre condition $\mathrm{I}^{\prime}$, the constant $\epsilon$ can be chosen so that $r(\xi, \eta, \zeta, \epsilon)>0$ when $|\eta|=1$ and $\zeta \neq 0$. We have accordingly $P(y ; \eta) \geqq \epsilon Q(y ; \eta)$ on $\mathscr{D}_{\infty}^{r^{*}}$ (and hence also on $D_{\infty}^{r}$ ) for all $\eta$ with norm $|\eta|=1$. Combining this result with that given in Theorem 9.2 one obtains Theorem 10.3.

12. Elliptic partial differential equations. Consider now an operator $A x$ on the space $\mathcal{F C}_{\mathrm{k}}^{n}(T)$ to $\mathcal{F C}_{0}^{q}(T)$ defined by

$$
\begin{aligned}
A^{\sigma} x=p_{\alpha}^{\sigma j}(t) x_{\alpha}^{j}(t) & +\int_{T} K_{\alpha}^{\sigma j}(t, s) x_{\alpha}^{j}(s) d s, \\
& |\alpha| \leqq k ; j=1, \cdots, n ; \sigma=1, \cdots, q .
\end{aligned}
$$


It is assumed that the functions $p_{\alpha}^{\sigma f}(t)$ are integrable on $T$, the functions $p_{\alpha}^{\sigma f}(t)$ with $|\alpha|=k$ are continuous on $\bar{T}$, and $K_{\alpha}^{\sigma f}(t, s)$ are square integrable on $T \times T$. The region $T$ is assumed to satisfy the conditions described in the paragraph preceding Theorem 6.1.

By the principal part $P x$ of $A x$ will be meant the operator

$$
P^{\sigma} x=p_{\alpha}^{\sigma j}(t) x_{\alpha}^{j}(t), \quad|\alpha|=k .
$$

The difference $C x=A x-P x$ is a compact operator from $\mathcal{F C}_{\mathbf{k}}^{n}$ to $\mathcal{F C}_{0}^{p}$, as can be seen by the use of Theorem 6.1.

The operator $A$ will be said to be elliptic in case at each point $t$ on the closure $\bar{T}$ of $T$

$$
p_{\alpha}^{\sigma j} \xi^{\alpha} \zeta^{j}=0, \quad|\alpha|=k
$$

holds for nonnull complex numbers $\zeta=\left(\zeta^{1}, \cdots, \zeta^{n}\right)$ and real numbers $\xi=\left(\xi_{1}, \cdots, \xi_{m}\right)$ only in case $\xi=(0, \cdots, 0)$. Here, as before, $\alpha=\left(\alpha_{1}, \cdots, \alpha_{m}\right)$ and $\xi^{\alpha}=\xi_{1}^{\alpha_{1}} \xi_{2}^{\alpha_{2}} \cdots \xi_{m}^{\alpha_{m}}$. Clearly, $A$ is elliptic if and only if its principal part is elliptic. If we set

$$
r_{\alpha \beta}^{h j}=p_{\alpha}^{\sigma h} p_{\beta}^{\sigma j},
$$

then the condition of ellipticity is equivalent to the Legendre condition $\mathrm{I}^{\prime}$ given by (8.3) for the quadratic form

$$
J(x)=\|A X\|^{2}
$$

Combining Theorems 4.1 and 8.1 we obtain the following

THEOREM 12.1. Let $\mathfrak{K}$ be the subclass of all functions $x$ in $\mathcal{F}_{\mathbf{k}}^{n}$ such that $x_{\alpha}^{j}=0,|\alpha|<k$ on the boundary of $T$. The necessary and sufficient condition that the operator $A$ restricted to $\mathcal{K}$ be of finite nullity and that its range be closed is that $A$ be elliptic on $T$.

This theorem states that if $A$ is elliptic and is restricted to $\nVdash$ the equations $A x=f$ have a solution in $\mathcal{K}$ for all $f$ orthogonal to the solutions of $A^{*} z=0$, where $A^{*}$ is the adjoint of $A$ restricted to $\nVdash$. The theorem is accordingly an existence theorem. Here and elsewhere we shall omit the discussion of special cases when it is appropriate to study conditions under which $A^{*} z=0$ has only the trivial solution.

In the next two theorems it will be assumed that the boundary is of class $Q^{k}$ and that each boundary point $t_{0}$ is of class $\mu=1$. Consider now a boundary point $t_{0}$ and suppose that the coordinates have been chosen so that the $t_{1}$-axis is normal to the boundary at $t_{0}$. The operator $A$ will be said to be $N$-elliptic 
at $t_{0}$ if the relation (12.3) holds at $t_{0}$ for complex numbers $(\xi, \zeta)$ with $\xi_{2}, \cdots, \xi_{m}$ real only in case either $\zeta=(0, \cdots, 0)$ or $\xi=(0, \cdots, 0)$.

TheOREM 12.2. Suppose the boundary of $T$ is of class $B^{k}$ and each boundary point is of index $\mu=1$. A necessary and sufficient condition that the operator $A$ on ${ }^{C_{\mathrm{k}}^{n}}$ be of finite nullity and that its range be closed is that $A$ be elliptic on $T$ and that $A$ be $N$-elliptic at each boundary point of $T$.

This result is a consequence of Theorems 4.1 and 8.5 since the condition of ellipticity and the condition of $N$-ellipticity at boundary points is equivalent to the condition III' $^{\prime}$ of Legendre for the quadratic form (12.4).

Consider now an elliptic differential equation $A$ and consider a boundary point $t_{0}$. Suppose that the coordinate axes have been chosen so that the $t_{1}$-axis is normal to the boundary at $t_{0}$. Given real numbers $\xi_{2}, \cdots, \xi_{m}$ and polynomials $Z^{j}\left(\xi_{1}\right)$ of $\xi_{1}$, which do not vanish simultaneously, then at $t_{0}$ the polynomials

$$
p_{\alpha}^{\sigma j} \xi Z^{j}, \quad|\alpha|=k,
$$

considered as polynomials of $\xi_{1}$ do not vanish simultaneously when $\xi_{1}$ is real, since $A$ is elliptic. However they may have common zeros which are complex. Let $\tau_{1}$ be the maximum number of these whose imaginary parts have like signs and let $\tau$ be the maximum of $\tau_{1}$ for all choices of real numbers $\xi_{2}, \cdots, \xi_{m}$ and all polynomials $Z^{j}\left(\xi_{1}\right)$ which do not vanish simultaneously. Let $r\left(t_{0}\right)$ be the smaller of $\tau$ and $k$. Let $B$ be the subclass of all $x$ in $\mathfrak{A}$ such that in a neighborhood of $t_{0}$ the relations $x_{\alpha}^{j}=0,|\alpha|<r\left(t_{0}\right)$ hold on the boundary of $T$. We have the following

Theorem 12.3. Suppose that the boundary of $T$ is of class $B^{k}$ and that each boundary point is of index $\mu=1$. If $A$ is elliptic, then the operator $A$ restricted to the subspace $B$ described above is of finite nullity and the range of $A$ is closed.

This result is an immediate consequence of Theorems 8.7 and 4.1 since the quadratic form $J(x)=\|A x\|^{2}$ satisfies the Legendre condition $\mathrm{V}^{\prime}$. It is clear that Theorems 12.1 and 12.2 are corollaries of Theorem 12.3.

13. Differentiability theorems. In the present section we shall be concerned with the system

$$
A^{\sigma} X=f^{\sigma} \quad(\sigma=1, \cdots, q)
$$

where $A^{\sigma}$ is given by (12.1). It will be assumed that the coefficients $p_{\alpha}^{\sigma f}(t)$ are of class $B^{(r)}$ on $T$ and that the coefficients $K_{\alpha}^{\sigma g}(t, s)$ are of class $D^{(r)}$ in $t$ on $T$ for almost all $s$ on $T$. The functions $K_{\alpha}^{\sigma j}(t, s)$ and its partial derivatives of orders $\leqq r$ with respect to $t$ are assumed to be square integrable on $T \times T$. Finally it is assumed that $f^{\sigma}$ is of class $D^{(r)}$ and that its partial derivatives of 
orders $\leqq r$ are square integrable on every interval in $T$. It will be convenient to say that a function having the properties ascribed to $f^{\sigma}$ is of class $D_{2}^{(r)}$ on $T$.

THEOREM 13.1. If $A$ is elliptic, $a$ solution $x$ of equations (13.1) is of class $D_{2}^{(\ell+r)}$ on $T$.

As will be seen from the proof, it is sufficient to consider the case when $r=1$. Moreover we can assume that (13.1) is the simpler form

$$
p_{\alpha}^{o j} x_{\alpha}^{j}=f^{\sigma}, \quad|\alpha|=k
$$

since the remaining terms can be incorporated in the functions $f^{\circ}$. Let $G$ be the gradient operator $D_{r}=i\left(\partial / \partial t_{\tau}\right)(\tau=1, \cdots, m)$. It is easily seen that the operator

$$
G A x=i p_{\alpha}^{\sigma j} \frac{\partial x_{\alpha}^{j}}{\partial t_{\tau}}+i \frac{\partial p_{\alpha}^{\sigma j}}{\partial t_{\tau}} x_{\alpha}^{j} \quad(\sigma=1, \cdots, q ; \tau=1, \cdots, m)
$$

is elliptic on $T$.

Consider now a point $t_{0}$ interior to $T$. By Theorem 7.3 there is a neighborhood $N$ of $t_{0}$ and an $\epsilon>0$ such that the inequality

$$
\|G A x\|^{2} \geqq \epsilon\|x\|_{k+1}^{2}
$$

holds for all $x$ in $\mathcal{H C}_{k+1}$ vanishing on the complement of $N$. We can assume that $N$ is an interval. Let $x$ be a solution of (13.2). Let $\rho(t)$ be a function of class $C^{\infty}$ whose support set is interior to $N$ and is equal to unity in a neighborhood of $t_{0}$. We shall prove that $y^{j}=\rho x^{i}$ is of class $D_{2}^{(k+1)}$. In the proof we can assume that $T$ coincides with $N$. Observe first that $y$ is a solution of an equation of the form

$$
p_{\alpha}^{\sigma j} y_{\alpha}^{j}=\dot{g},
$$

where $g^{\sigma}$ is of class $D_{2}^{(1)}$ and vanishes near the boundary of $T=N$. Let $y_{\alpha}^{\text {th }}$ and $g^{\boldsymbol{t} h}$ be the $h$-integral means (see $\$ 5$ ) of $y_{\alpha}^{j}$ and $g^{\sigma}$, where $h$ has been chosen so small that $y^{\text {jh }}$ is identically zero in a neighborhood of the boundary of $T$. Then

$$
p_{\alpha}^{\sigma j} y_{\alpha}^{j h}=\phi^{\sigma h}
$$

where

$$
\phi^{\sigma h}=g^{\sigma h}-\left[\left(p_{\alpha}^{\sigma j} y_{\alpha}^{j}\right)^{h}-p_{\alpha}^{\sigma j} y_{\alpha}^{j h}\right] .
$$

We have $\left(y^{j h}\right)_{\alpha}=(1-h)^{k} y_{\alpha}^{j h}$, by (5.6). Hence

$$
\left\|G A y^{k}\right\|^{2} \geqq \epsilon\left\|y^{k}\right\|_{k+1}^{2}
$$


by (13.3). Using Lemmas 5.1, 5.2 and (5.5) it is seen that $\phi^{\sigma h}, D_{\tau} \phi^{\sigma h}$ converge in the mean of order two to $g^{\sigma}, D_{\tau} g^{\sigma}$ respectively. It follows from (13.4) and (13.5) that $\left\|y^{h}\right\|_{k+1}$ is bounded and hence converges weakly to a function in $\mathfrak{F}_{k+1}$. This function must coincide with $y$. The original function $x$ is therefore of class $D_{2}^{k+1}$, as was to be proved.

THEOREM 13.2. Suppose that the boundary of $T$ is of class $B^{(k+r)}$ and each boundary point is of index $\mu=1$. If $A$ is elliptic and is $N$-elliptic on the boundary of $T$, then under the hypothesis given at the beginning of this section a solution $x$ of (13.1) is in the class $\mathfrak{H}_{k+r}$.

The proof is like that given for Theorem 13.1 with only minor modifications and will be omitted. It is in this proof that the special form of the integral mean is used.

14. Further differentiability theorems. The differentiability theorems given in the last sections are inadequate for problems in the calculus of variations. In variational problems we are concerned with solutions $y$ of system

$$
L(x)=J(x, y)
$$

where $L(x)$ is a linear functional, $J(x, y)$ is a bilinear functional and $x, y$ are restricted to lie in certain subspaces of the given Hilbert space. The differentiability theorems, as stated in the last section, do not apply unless $J$ is of the special form (12.4).

In the present section we shall assume that the Hilbert space with which we are concerned is the Hilbert space $\mathcal{C}_{k}^{n}(T)$ used in the preceding pages. We assume that $L(x)$ and $J(x, y)$ are of the form

$$
L_{k}(x)=\int_{T}^{x_{\alpha}^{h} J_{\alpha}^{h} d t, \quad|\alpha| \leqq k,}
$$

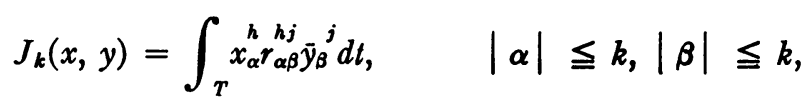

where the indices are those described in $\$ 6$.

We make the following assumptions:

(1) The functions $r_{\alpha \beta}^{h j}$ are essentially bounded on $T$ and the functions $f_{\alpha}^{h}$ are square integrable on $T$.

(2) The functions $r_{\alpha \beta}^{h j}$ with $|\alpha|=|\beta|=k$ are continuous on $T$, and there is a constant $\epsilon>0$ such that

$$
\operatorname{Re}\left(r_{\alpha \beta}^{h j} \xi^{\alpha+\beta} \zeta^{k} \bar{\zeta}^{j}\right) \geqq \epsilon|\xi|^{2 k}|\zeta|^{2}
$$

holds on $T$. 
(3) There is an integer $s \geqq 0$ such that, if we set $k_{0}=k-s+1$ when $k>s$, $k_{0}=0$ otherwise, then

(i) The coefficients $r_{\alpha \beta}^{i}\left(k_{0} \leqq|\alpha| \leqq k\right)$ are of class $B^{|\alpha|-k+\imath}$ on $T$.

(ii) The coefficients $f_{\alpha}^{h}\left(k_{0} \leqq|\alpha| \leqq k\right)$ are of class $D_{2}^{(k)}$ on $T$.

It should be noted that we do not require that the relation $i_{\alpha \beta}^{N}=r_{\beta \alpha}^{\text {J }}$ hold for all $\alpha, \beta$ as was done in the preceding pages.

THEOREM 14.1. Let $y$ be a function in $\mathfrak{H C}_{k}=\mathfrak{H C}_{k}^{n}(T)$ satisfying the conditions

$$
L_{k}(x)=J_{k}(x, y)
$$

for all $x$ in the class $\mathbb{e}$ of all $x$ of class $C^{\infty}$ whose support set is in $T$. Under the hypotheses made above, the function $y$ is of class $D_{2}^{k+s}$ on $T$.

As a first step in the proof of this result let $D_{\sigma}=i\left(\partial / \partial t_{\sigma}\right)(\sigma=1, \cdots, m)$ and set, for $k_{0} \leqq|\gamma| \leqq k, 0 \leqq|\delta|<k_{0}$

$$
\begin{aligned}
J_{k+1}(x, y) & =\int_{T} D_{\sigma} x_{\gamma}^{k}\left[r_{\gamma \beta}^{h j} \bar{D}_{\alpha} \bar{y}_{\beta}^{j}+\left(D_{\sigma} r_{\alpha \beta}^{h j}\right) y_{\beta}^{j}\right] d t-\int_{T} \Delta x_{\delta}^{h \gamma_{\delta \beta}} y_{\beta}^{j j} d t, \\
L_{k+1}(x) & =\int_{T} D_{\sigma} x_{\gamma}^{j} D_{\sigma} f_{\gamma}^{j} d t-\int_{T} \Delta x_{\delta}^{j} f_{\delta}^{j} d t .
\end{aligned}
$$

Here $\Delta x$ denotes the Laplacian of $x$.

LEMMA 14.1. If $y$ is in $\mathfrak{F C}_{k+1}$, then

$$
J_{k+1}(x, y)=-J_{k}(\Delta x, y), \quad L_{k+1}(x)=-L(\Delta x)
$$

for all $x$ in $\mathcal{C}$. If (13.3) holds for all $x$ in $\mathfrak{C}$ then

$$
L_{k+1}(x)=J_{k+1}(x, y)
$$

holds for all $x$ in $\mathrm{e}$. Moreover the functionals $J_{k+1}, L_{k+1}$ satisfy the conditions (1), (2), (3) of Theorem 14.1 with $k$ replaced by $k+1$ and s replaced by $s-1$.

The relations (14.5) follow from the divergence theorem. One uses the fact that $x$ is identically zero in a neighborhood of the boundary of $T$. The relation (14.6) follows from (14.3) and (14.5) and the fact that $\Delta x$ is in $e$ whenever $x$ is in $\mathfrak{e}$. The last statement in the theorem is readily verified.

CoRollary. If Theorem 14.1 holds when $s=1$, it holds for every positive integer $s$.

In view of this corollary we shall assume hereafter that $s=1$.

LEMMA 14.2. If $s=1$, one can assume that $r_{\alpha \beta}^{N} \equiv 0$ on T unless $|\alpha|=|\beta|=k$.

This follows because for $|\alpha|<h$ we can replace $f_{\alpha}^{h}$ by $f_{\alpha}^{h}-\bar{r}_{\alpha \beta}^{h j} y_{\beta}^{\prime}$ and $r_{\alpha \beta}^{h j}$ by 0 without altering our hypotheses on the coefficients. If $|\alpha|=k$, then our 
hypotheses imply that $f_{\alpha}^{h}-\bar{r}_{\alpha \beta}^{h y} y_{\beta}^{j}$ with $\beta$ summed subject to the restriction that $|\beta|<k$ is of class $D_{2}^{1}$ and hence can replace $f_{\alpha}^{h}$ provided that we replace $r_{\alpha \beta}^{h y}$ by 0 , as before. This proves the lemma.

Hereafter we shall assume that $r_{\alpha \beta}^{n j}=0$ unless $|\alpha|=|\beta|=k$.

LEMMA 14.3. If $s=1$ we can assume without loss of generality that $T$ is an interval, that $y$ and $f$ vanish identically on a neighborhood of the boundary of $T$, and that there is a constant $\epsilon>0$ such that the inequality

$$
\operatorname{Re} J_{k}(x, x) \geqq \epsilon\|x\|_{k}^{2}, \quad \operatorname{Re} J_{k+1}(x, x) \geqq \epsilon\|x\|_{k+1}^{2}
$$

holds for all $x$ in $\mathfrak{e}$.

In order to establish the differentiability of $y$ in a neighborhood of a point $t_{0}$ it is sufficient to establish the differentiability of $\rho y$, where $\rho$ is a function of class $C^{\infty}$ having $\rho=1$ in a neighborhood of $t_{0}$. In view of Theorem 7.2 applied to $J_{k}(x, x)+\bar{J}_{k}(x, x)$ and $J_{k+1}(x, x)+\bar{J}_{k+1}(x, x)$ we can select a neighborhood $N$ of $t_{0}$ such that for a suitable choice of $\epsilon$ the inequalities (14.7) hold for all functions $x$ of class $C^{\infty}$ whose support set is in $T$. This neighborhood $N$ may be chosen to be an interval with its center at $t_{0}$. Consider now a real function $\rho$ of class $C^{\infty}$ such that $\rho=1$ in a neighborhood of $t_{0}$ and whose support set is interior to $N$. Let $S$ be the points in $T$ at which $\rho(t) \equiv 0$. Then the equations

$$
I_{k}(\rho x, y)-L_{k}(\rho x)=0
$$

can be put in the form

$$
\int_{T}\left\{\rho x_{\alpha}^{k}\left[r_{\alpha \beta}^{h j} \bar{y}_{\beta}^{j}-\bar{f}_{\alpha}^{h}\right]-x_{\gamma}^{h} \bar{g}_{\gamma}^{h}\right\} d t=0
$$

where $\gamma$ is summed on the range $0 \leqq|\gamma|<k$. The functions $g_{\gamma}$ are of class $\mathscr{L}_{2}$ on $T$ and vanish identically on $S$. Setting $z=\rho y$ we see that

$$
z_{\alpha}^{h}=\rho y_{\alpha}^{h}+p_{\alpha}^{h}
$$

where $p_{\alpha}^{n}$ is of class $D_{2}^{1}$ on $T$ and $p_{\alpha}^{h} \equiv 0$ on $S$. Setting

$$
g_{\alpha}^{h}=f_{\alpha}^{h}-i_{\alpha \beta}^{h j} p_{\beta}^{j}, \quad|\alpha|=k,
$$

we obtain the relation

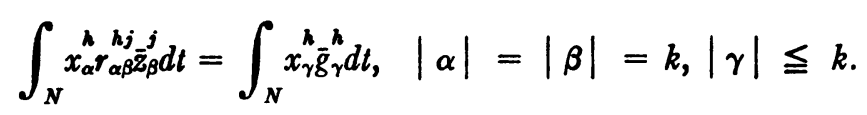

Replacing $T$ by $N, y$ by $z$ and $f$ by $g$ it is seen that the conditions imposed in Theorem 14.1 with $s=1$ still hold. This proves the lemma. 
In the next lemma we use the superscript $h$ to denote the $h$-integral mean of $y$, as described in $\$ 5$ and not as an index on the range $1, \cdots, n$ as used above. We shall use the notation $\mathcal{K}_{k}$ to be the subclass of all $x$ in $\mathfrak{K}_{k}$ such that $x_{\alpha}^{j}=0|\alpha|<k$ on the boundary of $T$.

LEMMA 14.4. There is a positive number $h_{0}$ such that the integral mean $y^{h}\left(0<h \leqq h_{0}\right)$ of $y$ is in $\mathcal{K}_{k+1}$ and satisfies a condition of the form

$$
J_{k+1}\left(x, y^{h}\right)=L_{k+1}^{h}(x)
$$

where $L_{k+1}^{n}$ are linear forms on $\mathfrak{K}_{k+1}$ such that

$$
\lim _{h=0} L_{k+1}^{h}(x)=L_{k+1}(x)
$$

for each $x$ in $\aleph_{k+1}$.

It is understood that the simplifications described in the preceding lemmas have been made. Consider now the formula

$$
\int_{T} x_{\alpha}^{i}(\rho) r_{\alpha \beta}^{i j}(\rho) \dot{y}_{\beta}^{j}(\rho) d \rho=\int_{T} x_{\gamma}^{i}(\rho) \bar{f}_{\gamma}^{i}(\rho) d \rho
$$

which holds for all $x$ in $e$. For small values of $h$ the substitution

$$
\rho=(1-h) t+h s
$$

where $s$ is restricted to $T$ can be made. Since $y$ vanishes in a neighborhood of the boundary of $T$ the condition (14.10) is equivalent to the condition that

$$
\int_{T} x_{\alpha}^{i}(t) r_{\alpha \beta}^{i j}(\rho) \bar{y}_{\beta}^{j}(\rho)(1-h)^{-|\alpha|} d t=\int_{T} x_{\gamma}^{i}(t) \vec{f}_{\gamma}^{i}(\rho)(1-h)^{-|\gamma|} d t
$$

for all $x$ in $\mathcal{C}$. Integrating with respect to $s$ over $T$ we obtain

$$
J_{k}\left(x, y^{h}\right)=L_{\dot{x}}^{h}(x)
$$

where

$$
\begin{array}{rlrl}
L_{k}^{h}(x) & =\int_{T} x_{\gamma}^{i}(t) \bar{F}_{\gamma}^{h i}(t) d t, & \\
F_{\gamma}^{h i}(t) & =(1-h)^{|\gamma|}\left(f_{\gamma}^{i}\right)^{h}-g_{\gamma}^{h i}, & g_{\delta}^{h i}=0,|\delta|<k, \\
g_{\alpha}^{h i} & =(1-h)^{-|\alpha|}\left[\left(r_{\alpha \beta}^{i j} y_{\beta}^{j}\right)^{h}-r_{\alpha \beta}^{i j}\left(y_{\beta}^{j}\right)^{h}\right], & & |\alpha|=k .
\end{array}
$$

Finally set, as in (13.4) with $|\alpha|=k,|\delta|<k$

$$
L_{k+1}^{h}(x)=\int_{T}\left[D_{\sigma} x_{\alpha}^{j} D_{\sigma} F_{\alpha}^{h j}-\Delta x_{\delta}^{j} F_{\delta}^{h j}\right] d t
$$


In view of Lemma 13.1 it follows that (13.8) holds. Applying the results described in $\$ 5$ and in particular that given in Theorem 5.5 it is seen that (14.9) holds.

LEMma 14.5. Given a linear form $L(x)$ on $\aleph_{k+1}$ there is a vector $z$ in $\aleph_{k+1}$ such that

$$
L(x)=J_{k+1}(x, z)
$$

holds for every $x$ in $\aleph_{k+1}$.

In view of the relations (14.7), this result is a corollary to Theorem 4.2.

We are now in position to complete the proof of Theorem 14.1. By virtue of the last lemma we may select $z$ in $\varkappa_{k+1}$ so that

$$
L_{k+1}(x)=J_{k+1}(x, z)
$$

for all $x$ in $\mathfrak{K}_{k+1}$. Combining this result with (14.10) we see that

$$
\lim _{h=0} J_{k+1}\left(x, y^{h}\right)=J_{k+1}(x, z)
$$

for every $x$ in $x_{k+1}$. It follows from Lemma 14.5 that $y^{h} \rightarrow z$ and consequently that $z=y$. The function $y$ is accordingly in $\varkappa_{k+1}$, as was to be proved.

\section{REFERENCES}

1. N. Aronszajn, On coercive integro-differential quadratic forms, Conference on Partial Differential Equations, University of Kansas, 1954, Technical Report No. 14, pp. 94-106.

2. F. E. Browder, On regularity properties of solutions of elliptic differential equations, Comm. Pure Appl. Math. vol. 9 (1956) pp. 351-361.

3. - Eigenfunction expansions for non-symmetric partial differential operators. II, Amer. J. Math. vol. 81 (1959) pp. 1-22.

4. - Estimates and existence theorems for elliptic boundary value problems, Proc. Nat. Acad. Sci. U.S.A. vol. 45 (1959) pp. 365-372.

5. J. W. Calkin, Functions of several variables and absolute continuity. I, Duke Math. J. vol. 6 (1940) pp. 170-186.

6. R. Dennemeyer, Quadratic forms in Hilbert space and second order elliptic differential equations, Ph.D. Dissertation, University of California, Los Angeles, 1956, 108 pp.

7. K. O. Friedrichs, On the differentiability of the solutions of linear elliptic differential equations, Comm. Pure Appl. Math. vol. 7 (1954) pp. 345-392.

8. L. Gaarding, Dirichlet's problem for linear elliptic partial differential equations, Math. Scand. vol. 1 (1953) pp. 55-72.

9. L. M. Graves, The Weierstrass condition for multiple integral problems, Duke Math. J. vol. 5 (1939) p. 656.

10. L. Hörmander, On the theory of general partial differential operators, Acta Math. vol. 94 (1955) pp. 161-284.

11. M. R. Hestenes, Applications of the theory of quadratic forms in Hilbert space to the calculus of variations, Pacific J. Math. vol. 1 (1951) pp. 525.

12. - Sufficient conditions for multiple integral problems in the calculus of variations, Amer. J. Math. vol. 70 (1948). 
13. F. John, Derivation of continuous weak solutions of linear elliptic differential equations, Comm. Pure Appl. Math. vol. 6 (1953) pp. 327-335.

14. P. Lax, On Cauchy's problem for hyperbolic equations and the differentiability of solutions of elliptic equations, Comm. Pure Appl. Math. vol. 8 (1955) pp. 615-633.

15. C. Morrey, Functions of several variables and absolute continuity, Duke Math. J. vol. 6 (1940) pp. 187-215.

16. - Multiple integral problems in the calculus of variations, University of California Publications in Mathematics, New Series, vol. 1 (1943).

17. - Second order elliptic systems of differential equations, Contribution to the theory of partial differential equations, Annals of Mathematics Studies, no. 33, Princeton University Press, 1954.

18. L. Nirenberg, Remarks on strongly elliptic partial differential equations, Comm. Pure Appl. Math. vol. 8 (1955) pp. 648-674.

19. $M$. Schechter, On estimating elliptic partial differential equations in the $L_{2}$ norm, Amer. J. Math. vol. 79 (1957) pp. 431-443.

20. - Coerciveness of linear partial differential equations for functions satisfying sero Dirichlet type data, Comm. Pure Appl. Math. vol. 11 (1958) pp. 153-174.

21. - Solution of the Dirichlet problem for equations not necessarily elliptic, Bull. Amer. Math. Soc. vol. 64 (1958) pp. 371-372.

22. - Integral inequalities for partial differential operators and functions satisfying general boundary conditions, Comm. Pure Appl. Math. vol. 12 (1959) pp. 37-66.

23. - General boundary value problems for elliptic partial differential equations, Bull. Amer. Math. Soc. vol. 65 (1959) pp. 70-72.

24. L. van Hove, Sur l'extension de la condition de Legendre du calcul des variations aux integrales multiples d plusieurs fonctions inconnues, Nederl. Akad. Wetensch. Indag. Math. vol. 9 (1947) pp. 3-8.

UNIVERSITY OF California, Los Angeles, California 